Linköping Studies in Science and Technology

No. 1802

\title{
Self-doped Conjugated Polyelectrolytes for Bioelectronics Applications
}

Erica Zeglio

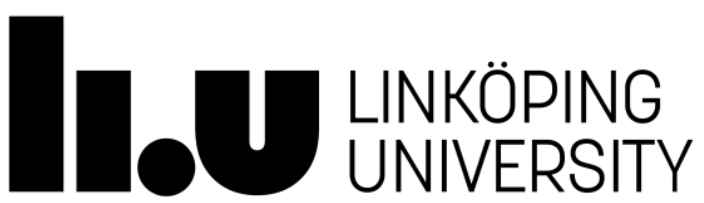

Biomolecular and Organic Electronics

Division of Applied Physics

Department of Physics, Chemistry and Biology

Linköping University, SE-581 83 Linköping, Sweden

Linköping 2016 
About the cover: conjugated polyelectrolytes interconnecting electronic transport with ionic transport across biomembranes.

Self-doped Conjugated Polyelectrolytes for Bioelectronics Applications

Erica Zeglio

During the course of the research underlying this thesis, Erica Zeglio was enrolled in Forum Scientium, a multidisciplinary doctoral programme at Linköping University, Sweden.

Copyright (C) Erica Zeglio, 2016, unless noted otherwise.

Printed in Sweden by Liu-Tryck, Linköping, Sweden 2016

Published articles and figures have been reprinted with the permission of the copyright holder.

ISBN: 978-91-7685-645-1

ISSN: 0345-7524

Linköping Studies in Science and Technology, Dissertation No. 1802 ii 
"Every chemistry student, faced by almost any treatise, should be aware that on one of those pages, perhaps in a single line, formula, or word, his future is written in indecipherable characters, which, however, will become clear "afterward": after success, error, or guilt, victory or defeat."

Primo Levi "The Periodic Table - Carbon" 


\section{Abstract}

Self-doped conjugated polyelectrolytes (CPEs) are a class of conducting polymers constituted of a $\pi$-conjugated backbone and charged side groups. The ionic groups provide the counterions needed to balance the charged species formed in the CPEs backbones upon oxidation. As a result, addition of external counterions is not required, and the CPEs can be defined as self-doped. The combination of their unique optical and electrical properties render them the perfect candidates for optoelectronic applications. Additionally, their "soft" nature provide for the mechanical compatibility necessary to interface with biological systems, rendering them promising materials for bioelectronics applications. CPEs solubility, aggregation state, and optoelectronic properties can be easily tuned by different means, such as blending or interaction with oppositely charged species, in order to produce materials with the desired properties. In this thesis both the strategies (blending and counterion exchange) have been explored to produce new functional materials that can be deposited to form a thin film and, therefore, used as an active layer in organic electrochemical transistors (OECTs). Microstructure formation of the films as well as influence on devices operation and performance have been investigated. We also show that these methods can be exploited to produce materials whose unique combination of self-doping ability and hydrophobicity allows incorporation into the phospholipid double layer of biomembranes, while retaining their properties. As a result, self-doped CPEs can be used both as sensing elements to probe the physical state of biomembranes, and as functional ones providing them with new functionalities, such as electrical conductivity. Integration of conductive electronic biomembranes into OECTs devices has brought us one step forward on the interface of man-made technologies with biological systems. 


\section{Populärvetenskaplig sammanfattning}

Självdopade konjugerade polymerer är en klass av elektroniska polymerer med en pi-konjugerad huvudkedja och laddade sidokedjor. Dessa laddade grupper utnyttjas då polymeren dopas till hög ledningsförmåga, och kompenserar injicerade elektroner eller hål. På grund av närvaron av laddade grupper behövs inga externa motjoner, och därför kan sådana polymerer kallas självdopade. Kombinationen av optiska och elektriska egenskaper gör konjugerade polyelektrolyter (CPE) lämpade för användning inom optoelektronik. Deras mekaniska egenskaper gör dem lämpliga för kombination med biologiska system, och de kan därför användas inom bioelektronik. Möjligheten att manipulera deras löslighet, aggregation och elektroniska struktur, i blandningar och med hjälp av joner eller ytaktiva substanser, kan ge många olika materialegenskaper. I avhandlingen beskrivs hur CPE's i blandningar och I kombinationer med ytaktiva ämnen kan forma tunna filmer som studeras i organiska elektrokemiska transistorer. Mikrostrukturens inverkan på transistorns funktion och prestanda har studerats. Vi har också visat hur kombinationen av CPE's och ytaktiva substanser ger självdopade och hydrofoba material som kan integreras i tunna bilager av fosfolipider, vilka bildar liposomer. På så sätt kan halvledande och lysande CPEs integrerade i biomembran av fosfolipider användas som sensorer för biomembraners struktur, och med metalliska CPEs ge biomembran elektronisk ledningsförmåga. Med hjälp av sådana strukturer har ytterligare ett steg tagits i integrationen mellan elektronik och biologiska strukturer. 
viii 


\section{Acknowledgements}

Nothing of this could have been accomplished without the support of the people around me. Each and everyone have contributed in his own and special way to the person and the researcher that I am today, and for that I am very thankful.

First of all I would like to thank my Family. Grazie Mamma e Papá. Grazie per il vostro costante incoraggiamento, anche quando le mie scelte sembrano un pó pazze. A huge thank goes to my husband Biswa. Thank you for sharing every single moment and being there for me to give me support and motivation when most needed. It would have been so much harder without you by my side. I would like to thank also my newly acquired family: Mom, Baba, Goutam and Subrata. I am glad you are part of my life.

Then I would like to deeply thank my supervisors. Thanks Olle for have given me the opportunity to be part of the Biorgel group. Thanks for your bright ideas and for the challenge to turn them into new and exciting results. Thanks Niclas for the many discussions on my data and for the help in finding the best way to convey my results to others.

Thanks a lot to my collaborators. I have learned so much from you. None of this would have been possible if you would not have shared your expertise and your knowledge with me. In particular thanks to Chiara, Mikhail, Sara, Johan, Jonas, Jens, Xuan, Ali, and Prof. Son. And thanks to Roger, Martina, and Prof. Thelakkat for their synthetic effort, which has been the foundation for my work.

Thanks to all the Forum members, and to the members of the Renaissance Network. It was very nice meeting you and share ideas as well as fun activities.

Thanks a lot to my officemates Fredrik, Deping and Luis, and to all the group members (you are so many!) for the fun group activities and always being there for a chat or a laugh.

Thanks Kicki for your warm welcome to Linkoping and Sarah for being such a nice flat mate and friend.

Thanks a lot to my Italian friends, here and at home. Grazie Mari, Davide, Ale, Sabri, Fede, Conci, e Monci: grazie per esserci sempre indipendentemente dalla distanza! Grazie a Tella per tutto l'aiuto da 
traslochi a matrimoni e Chimu e Frasa per avermi fatto sentire un po' meno distante da casa.

Thanks to Lorenza for have make me feel "from the warm south" for the first time in my life.

Thanks to all my friends over here. Especially thanks Camilla for all the nice chats, the "fatty" discussions, and for improving my Swedishness. Thanks Judit, for your contagious enthusiasm and the cakes. Thanks Pria for the nice breaks in though work days.

I would like to thank my flat mates Jeni, Claudia, and especially Vanessa. Thank you for putting up with me this last crazy year.

Thanks a lot also to all the friends in Lund. Especially thanks to Alice, Gibe, Neha, Chinu, Ranjana, Sandeep, Shubhu, and Erik for the nice chats and for been always there when needed. 


\section{List of Publications}

\section{Paper 1}

Conjugated Polyelectrolyte Blends for Electrochromic and Electrochemical Transistor Devices

Erica Zeglio, Mikhail Vagin, Chiara Musumeci, Fatima N. Ajjan, Roger Gabrielsson, Xuan T. Trinh, Nguyen T. Son, Ali Maziz, Niclas Solin, and Olle Inganäs*

Chemistry of Materials 27 (18), 6385-6393, 2015

\section{Paper 2}

Highly Stable Conjugated Polyelectrolytes for Water-based Hybrid Mode Electrochemical Transistors

Erica Zeglio, Jens Eriksson, Roger Gabrielsson, Niclas Solin, and Olle Inganäs*

Submitted

\section{Paper 3}

Conjugated Polyelectrolytes Blends for Highly Stable Accumulation Mode Electrochemical Transistors

Erica Zeglio, Martina M. Schmidt, Mukundan Thelakkat, Roger Gabrielsson, Niclas Solin, and Olle Inganäs*

Manuscript 


\section{Paper 4}

Electronic Polymers in Lipid Membranes

Patrik K. Johansson,* David Jullesson, Anders Elfwing, Sara I. Liin, Chiara Musumeci, Erica Zeglio, Fredrik Elinder, Niclas Solin and Olle Inganäs

Scientific Reports 5, 11242, 2015.

\section{Paper 5}

Conjugated Polyelectrolyte Blend as Photonic Probe of Biomembrane Organization

Erica Zeglio, Martina M. Schmidt, Mukundan Thelakkat, Roger Gabrielsson, Niclas Solin, and Olle Inganäs*

Chemistry Select 1 (14), 4340-4344, 2016

\section{Paper 6}

Electronic Membranes for Bioelectrochemical Transistor Devices

Erica Zeglio, Sara I. Liin, Fredrik Elinder, Jonas Christoffersson, Jens Eriksson, Deyu Tu, Martina M. Schmidt, Mukundan Thelakkat, Roger Gabrielsson, Niclas Solin, and Olle Inganäs*

Manuscript 


\section{Author Contributions}

\section{Paper 1}

Performed the experimental work together with other co-authors, except synthesis and atomic force microscopy measurements. Wrote the manuscript and contributed to final editing.

\section{Paper 2}

Participated in planning and performed all the experimental work, except conjugated polyelectrolytes synthesis and atomic force microscopy measurements. Wrote the manuscript and contributed to final editing.

\section{Paper 3}

Participated in planning and performed all the experimental work, except conjugated polyelectrolytes synthesis. Wrote the manuscript and contributed to final editing.

\section{Paper 4}

Performed emission and lifetime experiments. Participated to final editing of the manuscript together with other co-authors.

\section{Paper 5}

Participated in planning and performed all the experimental work, except conjugated polyelectrolytes synthesis. Wrote the manuscript and contributed to final editing. 


\section{Paper 6}

Participated in planning and performed most of the experimental work, except conjugated polyelectrolytes synthesis, atomic force microscopy measurements, oocytes handling and cells culture. Wrote the manuscript and contributed to final editing. 


\section{Abbreviations}

CP Conjugated polymer

CPE Conjugated polyelectrolyte

PEDOT-S Poly(4-(2,3-dihydrothieno[3,4-b]-[1,4]dioxin-2-ylmethoxy)-1-butanesulfonic acid)

PTEB-S Sodium poly(2-(3-thienyl)ethoxy-4-butylsulfonate)

PTHS Tetrabutylammonium poly(6-(thiophen-3-yl)hexane-1sulfonate)

DOPC 1,2-dioleoyl-sn-glycero-3-phosphocholine

POPC 1-palmitoyl-2-oleoyl-sn-glycero-3-phosphocholine

DPPC 1,2-dipalmitoyl-sn-glycero-3-phosphocholine

(Oct) ${ }_{2} \mathrm{NH}_{2} \mathrm{Cl}$ Dioctylammonium chloride

OECT Organic electrochemical transistor 


\section{Contents}

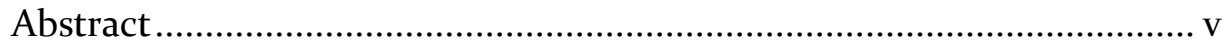

Populärvetenskaplig sammanfattning .................................................. vii

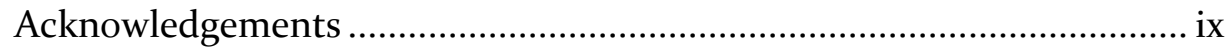

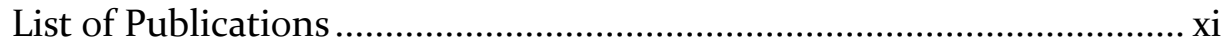

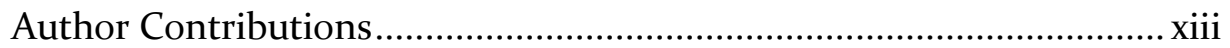

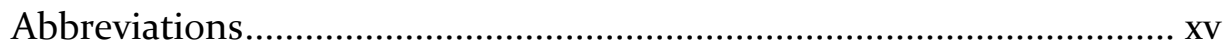

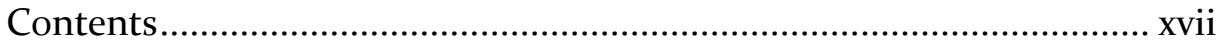

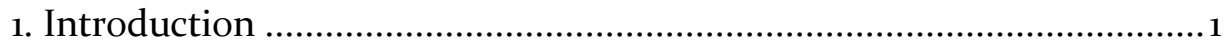

2. Conjugated Polymers .......................................................................... 3

2.1 Carbon - Conjugated Polymers Building Block............................. 4

2.2 Electronic Properties ..................................................................... 7

2.2.1 Interchain Charge Transport ........................................... 9

2.3 Conjugated Polyelectrolytes..........................................................10

2.4 Self-Doped Conjugated Polyelectrolytes .............................................11

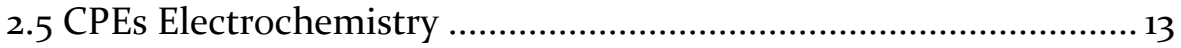

2.6 Optical Properties .......................................................................... 15

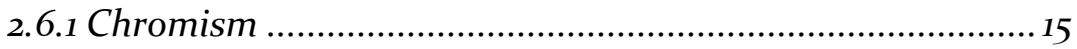

2.6.2 Emission Properties....................................................... 18

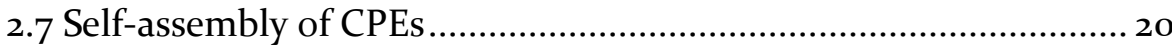

2.7.1 Aggregation of CPEs in Solution.......................................... 20

2.7.2 Tuning CPEs Solubility by Counterion Exchange ..................21

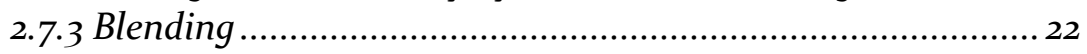

2.8 Film Formation .........................................................................23

2.8.1 Drying Films Characterization................................................ 24

2.8.2 Film Characterization ........................................................ 25

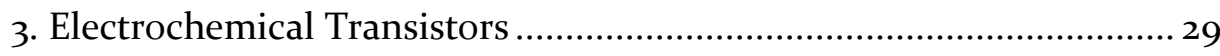

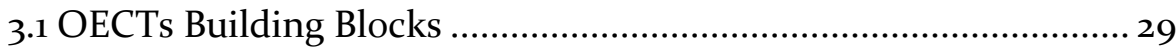


3.2 OECTs Characterization ................................................................. 30

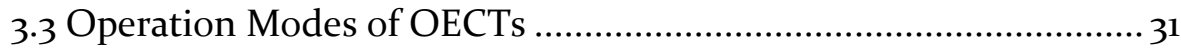

3.3.1 Tuning OECTs Operation Mode ........................................... 33

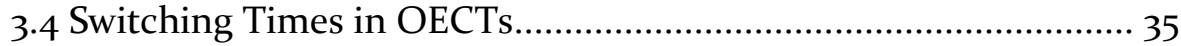

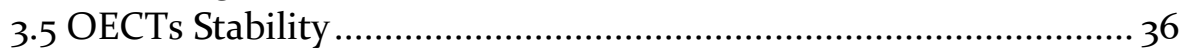

3.5.1 Stability towards Cycling ...................................................36

3.5.2 Stability in Water-based Electrolytes................................. 38

3.6 OECTs and Bioelectronics.............................................................. 39

4. Electronic Membranes ................................................................... 41

4.1 Biomembranes Constituents ...........................................................41

4.1.1 Phospholipids - Biomembranes Building Blocks .................. 41

4.1.2 Cholesterol - Regulator of Biomembrane Fluidity ................ 44

4.2 Probes for Membranes Physical State .............................................46

4.3 Conjugated Polyelectrolytes as Membrane Probes ......................... 47

4.4 Biomembranes and Ion Transport.................................................48 48

4.5 CPEs as an Interface to Ion Channels ..........................................49

5. Bioelectrochemical Transistor........................................................... 51

5.1 Biomembranes and Cells in OECTs................................................ 51

5.2 Preparation of Supported Lipid Bilayers (SLBs) ........................... 52

5.3 Electronic Membranes.............................................................. 53

5.4 A Bioelectrochemical Transistor .................................................. 55

5.4.1 Monitor Ion Channels in Oocytes........................................56

6. Conclusions and Future Outlook ......................................................... 59

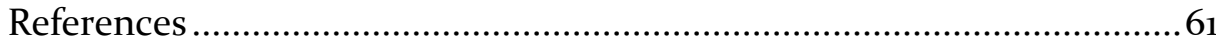




\section{Chapter 1}

\section{Introduction}

Organic electronics is a field of research concerning the use of conductive organic materials for the production of electronic devices. One of the technological developments introduced by this field is the manufacturing of low-cost devices using printing techniques. This has been rendered possible by the easy processability of organic materials from common solvents. Nowadays many electronic components can be printed and are already found on the market. However, a limit is represented by the lower efficiencies of organic materials with respect to their inorganic counterparts. More research efforts are, therefore, needed to improve existing technologies or introduce new ones. Research in organic electronics comprise many aspects, such as the development of new organic materials, understanding the processes behind device function, and introduction of new fabrication strategies. Therefore, combined efforts are required from the worlds of chemistry, physics and engineering.

Organic electronics at its early stages didn't include the field of biology. In the past decade, though, an idea developed hundreds years ago could be revived leading to the encounter of the fields of organic electronics and biology. In the middle 1750, an Italian scientist, Luigi Galvani, performed the first experiments in animal electricity, by connecting nerves of frog legs to metal wires. Stimulation by an electrical impulse lead to muscles contraction, and represented the first evidence of bioelectricity. In the present days, the advances obtained in the field of organic electronics could be exploited to develop new interfaces with biological system.

Bioelectronics takes advantage of the mixed ionic/electronic conductivity of organic materials to interface the world of the living, where messages are exchanged via ions, with electronics, where signals are exchanged via electrons. The soft nature of these materials enables a better match to the softness of tissues and cells with respect to their inorganic counterparts. This work has been focused on a special class of organic materials called "conjugated polyelectrolytes" (CPEs). In the first part of this thesis I will introduce you to the world of conjugated polyelectrolytes: what they are made of and which are their properties. Then, I will demonstrate that tuning CPEs properties from solution via self-assembly is an effective 
method to formulate new materials with desired properties. These properties have been exploited in chapter 3 for the fabrication of high performance devices in form of organic electrochemical transistors. In chapter 4 I will demonstrate that the same strategies can be used to produce materials with required properties for integration with biological systems. The molecular nature of CPEs has been exploited to create interfaces with bio-systems and tune their functions at the nanoscale. Then, in the last chapter, I will show that the two different concepts (CPEs for devices and CPEs for bio-related applications) can be reunited to lead to the production of original and exciting ways to interconnect electronics with biology. 


\section{Chapter 2}

\section{Conjugated Polymers}

This chapter is dedicated to a sub-class of polymers, called conjugated polymers. The word "polymer" derives from the Greek poly-, "many" and mer, "parts", as they are molecules composed by many repeating units linked together by covalent bonds.

Depending on their origin, polymers can be classified into natural or synthetic. Natural polymers are commonly synthesized by living organisms; some examples are silk, DNA, and cellulose. Synthetic polymers are synthesized by mankind through organic synthesis strategies. Due to their tunable physical and chemical properties, and simple processability, synthetic polymers have found great use in our everyday life. Moreover, their mechanical properties, such as softness and flexibility, can be adjusted according to the specific application. All these properties, combined with the possibility to render them biodegradable, make them the perfect candidates for healthcare and biomedical applications. ${ }^{1,2}$ Conjugated polymers are synthetic polymers possessing alternating single and double bonds in their chemical structure. They share all the above described properties with one interesting addition: electrical conductivity. ${ }^{3}$ This property has been exploited for several applications, from production of devices able to harvest solar energy (solar cells) ${ }^{4}$ to devices able to produce light (organic light emitting diodes) ${ }^{5}$ or even communicate with biological systems (electrochemical transistors). ${ }^{6}$ However, conjugated polymers cannot be electrically conductive if they are not doped, meaning that electrons shall be extracted or injected into their backbone to produce charge carriers and achieve conductivity. As we will see, the molecular structure of conjugated polymers plays a significant role in their ability to conduct charges. CPs molecular structure is mainly composed by carbon and hydrogen atoms with the addition of other elements, such as oxygen, sulfur and nitrogen, which are present in a lower extent. 


\subsection{Carbon - Conjugated Polymers Building Block}

The most important constituent of polymers is a chemical element belonging to the $14^{\text {th }}$ group of the periodic table: carbon. This element is so significant that chemists decided to devote to it an entire branch of chemistry, named "organic chemistry". The name "organic" date back to the time when scientists used to believe that compounds could be classified as organic or inorganic, depending on whether they were extracted from living matter or minerals, respectively. Even though today many organic compounds can be synthesized, the appellative remained unchanged.

Carbon is a nonmetal having electronic configuration $1 s^{2} 2 s^{2} 2 p^{2}$. The electronic configuration of an atom determines the distribution of the electrons around the nucleus, and plays a central role in atom reactivity and molecules formation. Electrons are arranged around the nucleus in atomic orbitals, which are wave functions $\psi$ describing the state of the electron. Through the value $\psi^{2}$, they define the probability to find an electron in the space. Atomic orbitals are classified by three quantum numbers. The principal quantum number $n$ defines the energy of the electrons and it can assume integers $n=1,2,3 \ldots$ The orbital angular momentum quantum number $l$ describes the shape of the orbitals $(l=0$, $1 . . n-1)$, while the magnetic quantum number $m_{l}$ describes the possible orientations of a certain orbital $\left(m_{l}=0, \pm 1 \ldots \pm l\right)$. One more quantum number, called spin quantum number $m_{s}$, describes electrons quantum state and can assume values $-1 / 2$ or $+1 / 2$. The electronic distribution into atomic orbitals is described by Pauli Exclusion Principle, which states that "only two electrons can occupy an atomic orbital, and to do so they must have opposite spins". Therefore the electronic configuration of carbon would be as depicted in Figure 2.1.

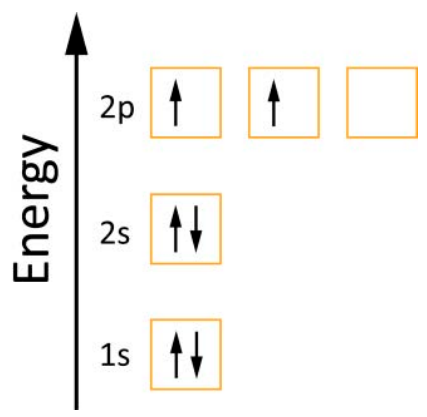

Figure 2.1 Electronic configuration of a carbon atom. 
Electrons possess same charge, therefore, due to repulsive forces, $\mathrm{p}$ electrons occupy two different degenerate $2 p$ orbitals, as described by Hund's rule. The particular electronic structure of an atom determines its possibility to form chemical bonds with other atoms. A covalent bond is a chemical bond that forms when two atoms share an electron pair. To do so, the atomic orbital of an atom should overlap with the one of another atom, each of them containing a single electron. This will lead to the creation of a molecular orbital shared between both the atoms and containing the two electrons. Considering the above described electronic structure, it would be expected that carbon can form only two bonds, as only two single electrons are available. However, this is not the case. Atomic orbitals can, in fact, superimpose to form hybrid orbitals. The total energy of the newly formed orbitals is lower with respect to the energy of the originals orbitals. Depending on the number of orbitals involved in the hybridization process, carbon can have hybridization $\mathrm{sp}^{3}$, $\mathrm{sp}^{2}$ or sp (Figure 2.2).
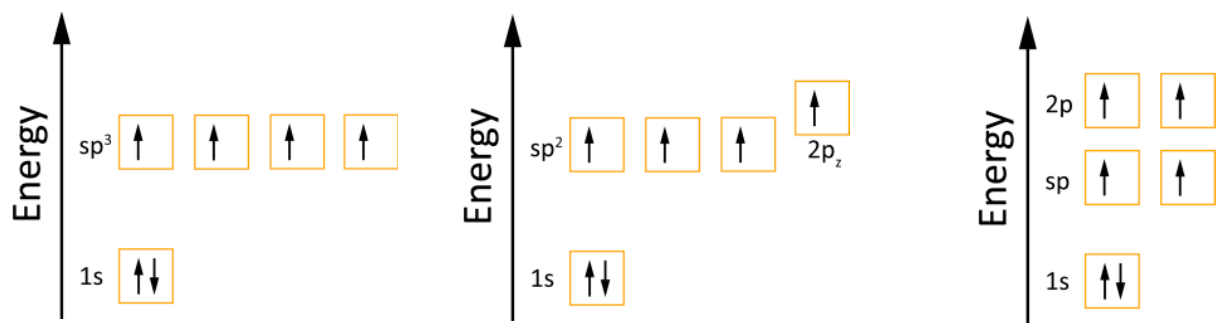

Figure 2.2 Electronic configuration of $\mathrm{sp}^{3} . \mathrm{sp}^{2}$, and sp hybrid orbitals of a carbon atom.

Consequently, depending on its hybridization, carbon will be able to form four, three or two covalent bonds with a corresponding number of atoms. The newly formed molecular orbitals will arrange in space in a way that minimizes the electronic repulsion, which means as far away from each other as possible (Figure 2.3).

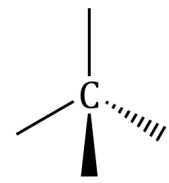

$\mathrm{sp}^{3}$<smiles>C=C(C)C</smiles>

$\mathrm{sp}^{2}$

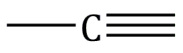

$\mathrm{sp}$

Figure 2.3 Possible hybridization geometries of a carbon atom. 
When the two atomic orbitals overlap on the line joining the two nuclei, the resulting molecular orbital is called sigma ( $\boldsymbol{\sigma}$ bond). Carbon atoms form $\sigma$ bonds with hybrid orbitals. When the overlapping between the two atomic orbitals occurs perpendicularly with respect to the line joining the nuclei, the formed molecular orbital is called pi ( $\pi$ bond). Carbon atoms form $\pi$ bonds with p orbitals. For example, in the molecule of ethylene (Figure 2.4) two carbon atoms are bonded together by a $\sigma$ bond and a $\pi$ bond. The electronic structure of a molecule can be predicted by a method called linear combination of atomic orbitals (LCAO). For each bond two molecular orbitals will be formed: a bonding orbital, having low energy, and an antibonding orbital. The latter, if occupied, leads to a reduction of cohesion forces between the atoms. From the diagram it can be also noticed that the $\pi$ bond is weaker (possess higher energy) than the $\sigma$ bond, due to less overlap of atomic orbitals.
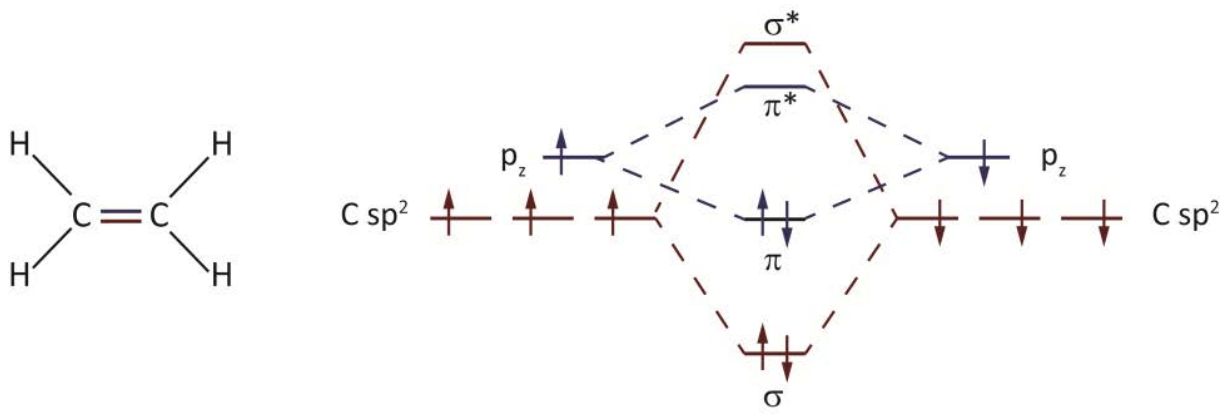

Figure 2.4 Chemical structure of ethylene molecule and corresponding molecular orbital diagram.

The $\mathrm{C}=\mathrm{C}$ distance in ethylene $(1.34 \AA$ ) is lower with respect to the C-C distance in ethane (1.55 $\AA$ ), as the double bond held the two atoms more closely than the single bond. ${ }^{7}$ Another important difference is that, despite $\pi$ bond, $\sigma$ bond allows free rotation of the atoms around the bond axis. Rotation of the atoms bonded by a $\pi$ bond will, in fact, preclude overlap between the atomic $\mathrm{p}$ orbitals and lead to bond breakage. The molecule of ethylene is the simplest member of a family of molecules called alkenes or olefins, which distinguishing feature is the presence of double bonds in their chemical structure.

Conjugated polymers (CPs) are polymers possessing alternating single $(\sigma)$ and double $(\pi)$ bonds in their backbone. One of the first representatives of this class of polymers is linear polyacetylene, which was synthesized for the first time in 1958. The chemical reaction involved olefins as a precursor and the use of a novel metal-based catalyst, for which the two scientists Karl Ziegler and Giulio Natta won the Nobel Prize 
in 1963. However, only in 1977, when Heeger, McDiarmid and Shirakawa demonstrated that exposures of polyacetylene films to halogens vapors increased their conductivity $10^{9}$ times, the potential of CPs was revealed. The discovery was awarded the Nobel Prize in 2000 and resulted in a paradigm shift from the idea of plastic as insulating or low conductive material to the new concept of highly conductive plastic for uses in electronics. The new field of organic electronics was born.

\subsection{Electronic Properties}

Conjugated molecules like polyacetylene can be described by structures having same nuclear configuration, but different electronic configurations. The real structure is a hybrid between all the possible structures and it is called resonance hybrid of the molecule. The total energy of the hybrid is lower than the energy of the contributing structures, due to electrons delocalization through the whole conjugated chain (Figure 2.5).

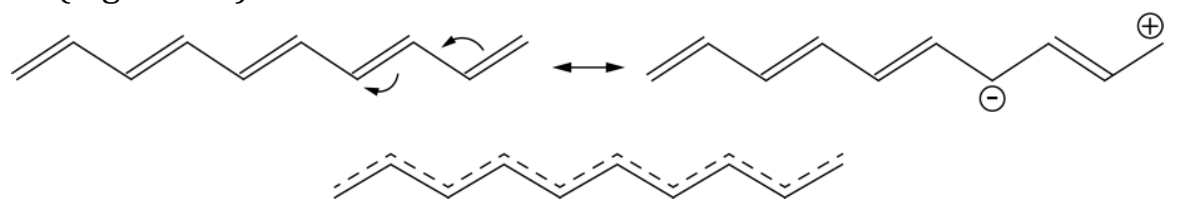

Figure 2.5 Two resonance forms and resonance hybrid of polyacetylene.

When only few monomer units $(<10)$ are connected to each other the molecule is termed oligomer, where oligo- derives from the greek "few". Increase of number of monomers in the conjugated chain leads to an increase of atomic orbitals with slightly dissimilar energy overlapping to form molecular orbitals. As a result, two bands will form: a valence band, formed by overlapping of bonding orbitals, and a conduction band, formed by overlapping of antibonding orbitals (Figure 2.6). 


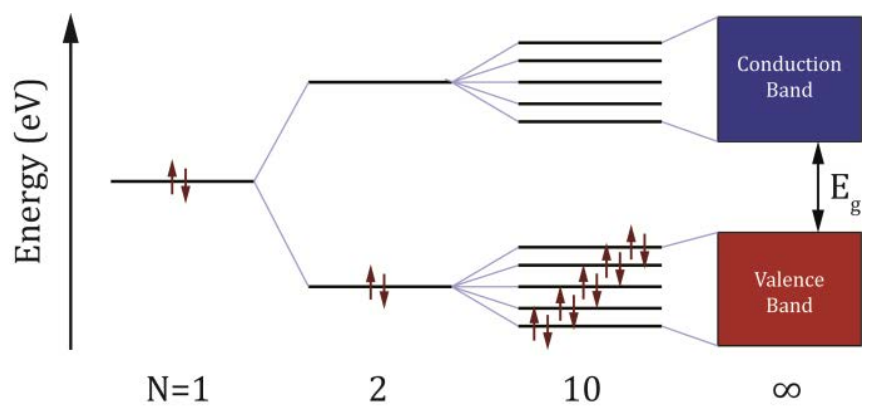

Figure 2.6 Formation of bands in conjugated polymers. $\mathrm{N}=$ number of interacting units.

The difference in energy between the highest occupied molecular orbital (HOMO) and lowest unoccupied molecular orbital (LUMO) determines the polymer band gap $\left(E_{g}\right)$. Depending on the magnitude of the band gap, materials are generally classified as insulator, semiconductor or conductor. CPs, like the above mentioned polyacetylene, are insulators, meaning that the band gap is too high and electrons cannot be promoted to the conduction band. However, they can be rendered conductive through doping.

There are two main forms of doping. N-type doping occurs when the neutral chain of a polymer is easily reduced to form carbanions, which charge is compensated by the insertion of cations into the polymeric matrix. P-type doping, instead, occurs when the neutral polymeric chain is easily oxidized to form carbocations, which charge in compensated by anions insertion into the polymeric matrix. The doping process can be accomplished in different ways. Chemical doping involves the addition of molecules that can act as dopant, meaning that they act as electronacceptor or electron-donor and remove or give electrons to the polymer backbone to form the so-called charge carriers. Another approach is to dope a polymer electrochemically: in this case, a potential difference generated between two electrodes leads to $\mathrm{CP}$ oxidation/reduction, and formed charges are compensated by ions from the electrolyte, as it will be shown in section 2.5 .

This thesis mainly focuses on p-type CPs. For these materials the main charge carriers are radical cations or di-cations. In the world of physics, a radical cation delocalized over a polymer segment is called polaron, while a delocalized di-cation is called bipolaron. Those terms take into account also the lattice distortion caused by the presence of the charges into the polymer backbone. ${ }^{8}$ The first charge carriers that form at low doping levels are polarons. Being radical cations, they possess spin $1 / 2$ and, therefore, their electronic signature can be studied using electron spin 
resonance spectroscopy (ESR), as shown in Paper $1 .{ }^{9}$ When doping level increases to intermediate doping the ESR signal starts to decrease, due to recombination of polarons to form bipolarons, which are spinless. Further increase in doping levels results in overlap between the bipolaron energy levels to form bipolaron bands (Figure 2.7).

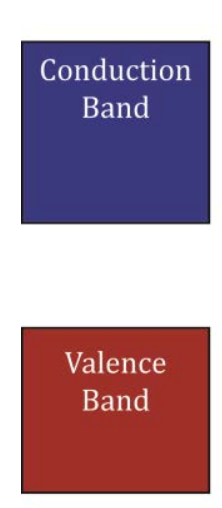

a)
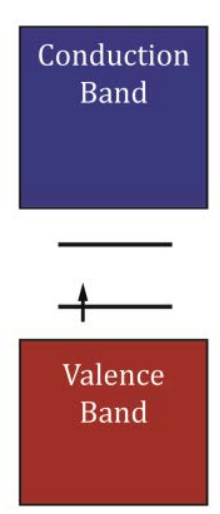

b)

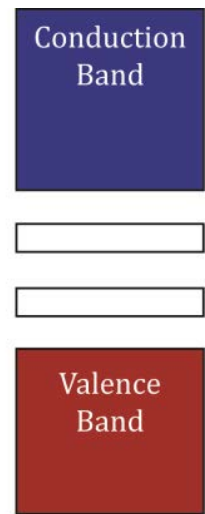

c)

Figure 2.7 Band structure of a p-type conjugated polymer a) undoped, b) in a polaron state, c) in a bipolaron state.

\subsubsection{Interchain Charge Transport}

The above described band theory relates to transport of charges between monomers of the same CP chain. However, conduction at the macroscale is not only determined by intrachain transport, but also by the ability of charge carriers to move from one $\mathrm{CP}$ chain to another one. In contrast with inorganic materials, where conduction is based on charges delocalization through the lattice, in CPs interchain charge transport occurs via "hopping" (Figure 2.8). ${ }^{10}$ 


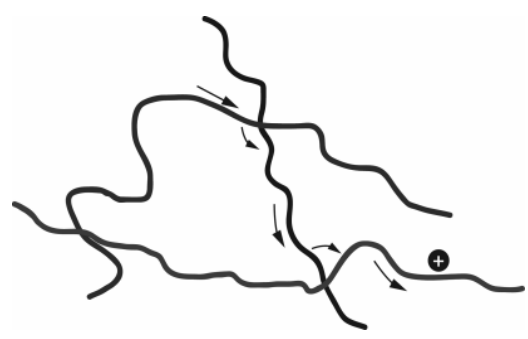

Figure 2.8 Transfer of a charge carrier between CPs chains.

This process possesses an activation energy and it is, therefore, temperature dependent: higher the temperature, larger the hopping probability. ${ }^{11}$ Moreover, factors affecting chains organization, such as selfassembly or aggregation phenomena, are of importance in the ability of charges to travel between CPs chains. ${ }^{12}$ As we will see in section 2.8, the supramolecular interactions occurring between CPs chains in solution affect the morphology of the deposited films: e.g. if the CPs chains form aggregates in solution, casting on a substrate results in a thin film made of large domains.

\subsection{Conjugated Polyelectrolytes}

The main disadvantage of the first generations of CPs was their poor solubility, which rendered them difficult to process. For example, polythiophene possesses extremely low solubility. Synthetic strategies have therefore been employed to improve CPs solubility and processability. Synthesis of derivatives comprising side chains covalently bonded to the $\pi$-conjugated backbone allows dissolution in organic solvents, due to increase of dissolution entropy. One of the most relevant examples is poly(3-hexylthiophene-2,5-diyl) (P3HT). However, devices development has rendered desirable CPs processability from polar and environmentally friendly solvents, such as water. Perhaps the most successful example of high performance water soluble material is the polymeric dispersion poly(3,4-ethylenedioxythiophene):polystyrene sulfonate PEDOT:PSS (Figure 2.9). 


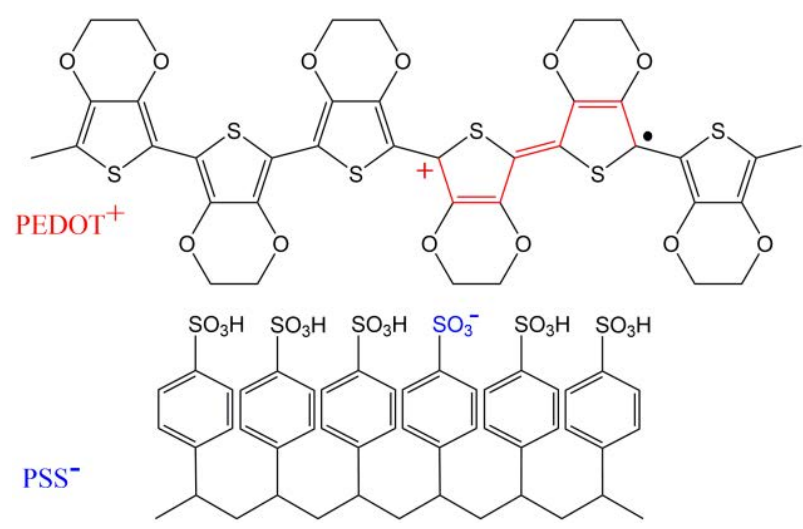

Figure 2.9 Chemical structure of PEDOT:PSS.

While PEDOT alone shows poor solubility, complexation with PSS leads to a material with high water solubility. Moreover, PSS provides sulfonate groups able to stabilize positive charges formed on the PEDOT backbone upon doping, leading to a material with high conductivity, above 1000 $\mathrm{S} / \mathrm{cm}^{13}$ Another possible approach consists in synthesizing CPs having electrolyte groups covalently attached to their backbone: the so-called conjugated polyelectrolytes (CPEs). CPEs can be divided into two main groups depending on the net charge of their side groups: anionic and cationic. This thesis mainly concern work on anionic conjugated polyelectrolytes, with a special focus on self-doped conjugated polyelectrolytes.

\subsection{Self-doped Conjugated Polyelectrolytes}

The concept of "self-doping" was firstly reported in 1987 by Wudl et al. as a method to improve the doping kinetics. ${ }^{14}$ In fact, the doping process involves diffusion of charged species to balance charges formed upon oxidation/reduction of the polymer backbone. In self-doped CPEs the potential counterions are readily available, as they are covalently bonded to the backbone. Three different kinds of self-doped CPEs have been used in this thesis: PEDOT-S, PTEB-S, and PTHS (Figure 2.10). 


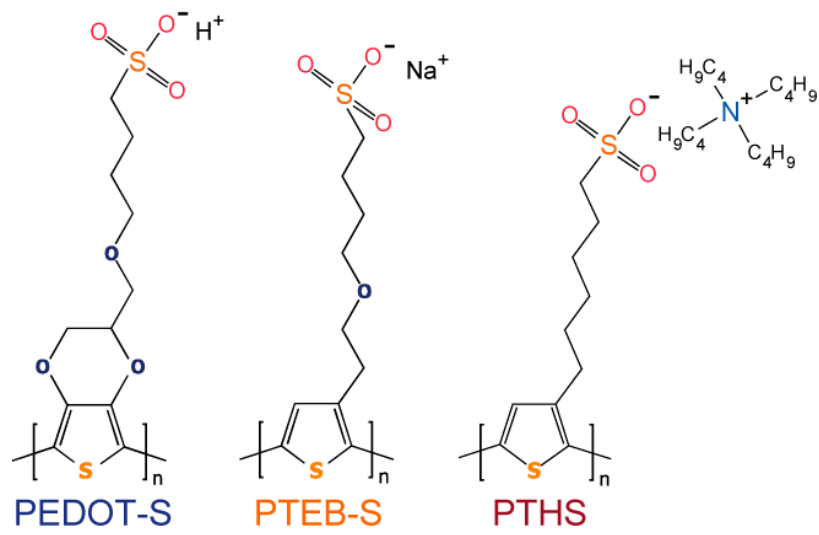

Figure 2.10 Chemical structure of the self-doped conjugated polyelectrolytes used in this thesis.

The presence of sulfonate groups covalently attached to their backbone guarantees them high water solubility. Moreover, the acid-base equilibria of these groups determines existence of the CPEs in an acid or conjugated base form. PEDOT-S exists in a protonated form in its pristine state, while PTEB-S and PTHS are found in a salt form. The acid-base equilibria of CPEs can be shifted by varying the $\mathrm{pH}$ of the solution, by addition of acids or bases. A decrease in $\mathrm{pH}$ will lead to a particular kind of chemical doping, called acid doping; where $\mathrm{H}^{+}$act as a dopant, by protonating the CPEs backbone. ${ }^{15}$ As a consequence, the doping level of CPEs can be tuned by $\mathrm{pH}$, as we will see for PEDOT-S in section 2.6.1. Sulfonate groups are negatively charged and, thus, able to stabilize formation of positive charges in the backbone upon oxidation. These CPEs are, therefore, p-type materials. However, structural differences in CPEs backbones determine dissimilar behaviour toward oxidation. PEDOT-S is found in a metallic, highly conductive state, in its pristine form, while PTEB-S and PTHS exist in a semiconducting state. This is due to the presence of two oxygens in PEDOT-S backbone, whose lone pairs can stabilize the positive charges formed in the conjugated backbone by electron donating effect. ${ }^{16}$ Another structural difference is represented by the number of monomer units. PEDOT-S is made of $\sim 12$ monomer units and, due to repulsion of negative charges of sulfonate groups, a single PEDOT-S chain is likely to assume a rod-like conformation. PTEB-S and PTHS possess $\sim 3500$ and $\sim 75$ monomer units, respectively. Therefore, single chains of these two CPEs in a good solvent are likely to assume a coiled conformation (Figure 2.11). 


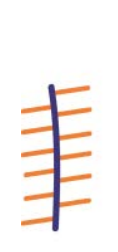

a)

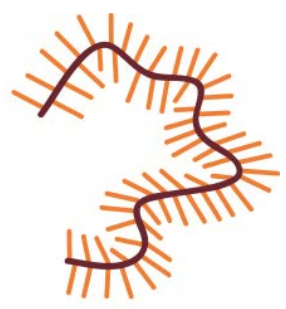

b)

Figure 2.11 Possible conformations adopted by single molecules of a) PEDOT-S and b) PTHS or PTEB-S chains in a good solvent.

\subsection{CPEs Electrochemistry}

The redox states of CPEs can be conveniently modified by means of electrochemistry. Here, the redox equilibria of a redox species is altered by application of a potential difference between a working electrode (W.E.) and a counter electrode (C.E), which are brought in contact by means of an electrolyte. A reference electrode (R.E.) is frequently used in order to quantify potentials of the electrochemical cell. In this thesis, two techniques have been used for CPEs characterization: cyclic voltammetry and four-terminals electrochemical setup (Figure 2.12).

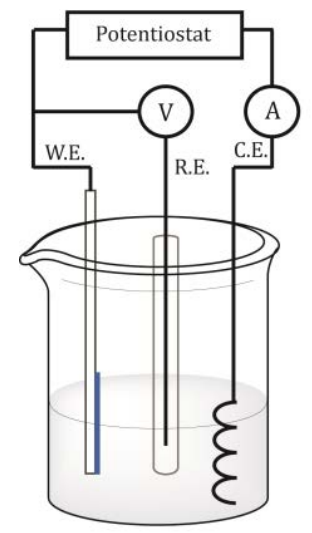

a)

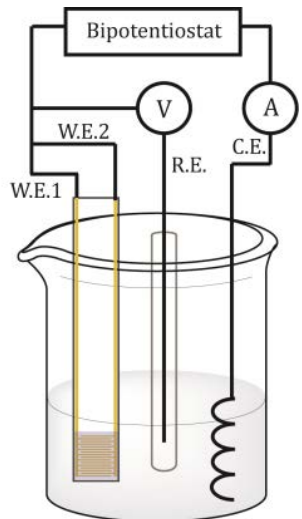

b)

Figure 2.12 Schematic representation of a) cyclic voltammetry and b) 4-terminals electrochemical setups used in this thesis. The sample is constituted by a polyelectrolyte film casted on top of an indium tin oxide (ITO) working electrode or gold interdigitated microelectrodes array (IMEA) on glass. 
In cyclic voltammetry (CV) potential sweeps lead to a current response generated by the doping/de-doping of the active material. Voltammetry response of PEDOT-S films shows a large capacitive behavior (Figure 2.13 , a). An anodic peak occurs at $+0.2 \mathrm{~V}$ while, in the reverse scan, a cathodic peak is found at $-0.03 \mathrm{~V}$. PTEB-S, instead, show an indistinct anodic process due to film oxidation and a cathodic band at $+0.55 \mathrm{~V}$ (Figure $2.13, \mathrm{~b}$ ). It can also be noticed that the PEDOT-S oxidation potential is much lower than PTEB-S. As mentioned in section 2.4, this is due to the structural differences in CPEs backbones. ${ }^{16}$
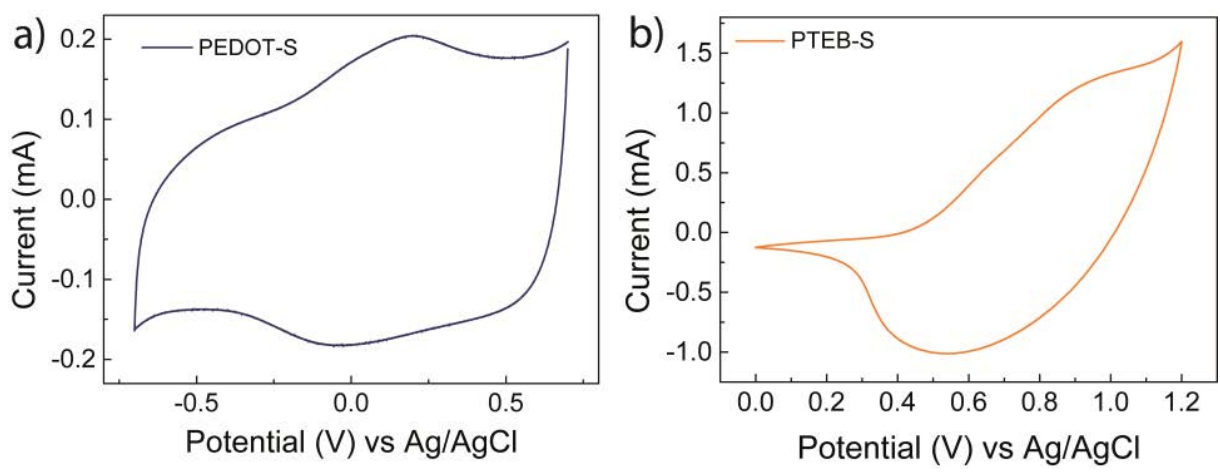

Figure 2.13 Cyclic voltammetry responses of a) PEDOT-S and b) PTEB-S films drop casted on ITO-coated substrates, measured in $\mathrm{LiClO}_{4}\left(0.1 \mathrm{M}\right.$ in $\left.\mathrm{CH}_{3} \mathrm{CN}\right)$ at $0.05 \mathrm{~V} / \mathrm{s}$ scan rate.

In the case of the four-terminals electrochemical setup, a small potential difference is applied between two working electrodes (W.E.1 and W.E.2) connected by means of a CPE/CP film. The voltammetric response is dominated by the currents flowing into the channel. If the measured currents are divided by the applied bias, it is possible to derive a conductance response as function of the applied potential (Figure 2.14) ${ }^{17}$ As formation of charge carriers is determined by CPE oxidation, the conductance response of the CPE film is governed by the oxidation potential of the CPE. For example, PEDOT-S shows a single sigmoidal response starting at $\mathrm{E}=-0.3 \mathrm{~V}$. PTHS, instead, exhibits a rise in conductance at $E=0.35 \mathrm{~V}$. Like PTEB-S, in fact, it possesses a semiconducting nature and, therefore, it is oxidized at higher potentials. 


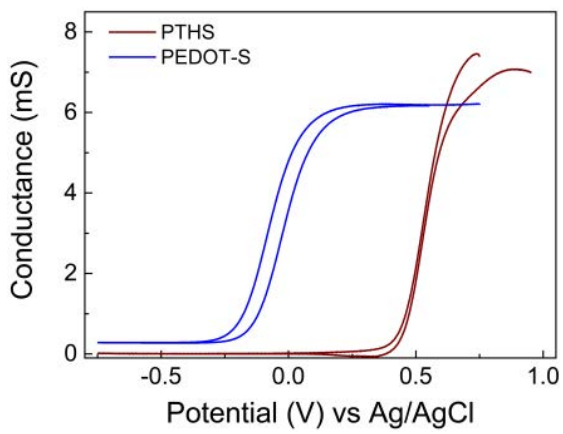

Figure 2.14 Conductance responses of PEDOT-S and PTHS, acquired for voltage bias of $0.05 \mathrm{~V}$ using a four-terminals electrochemical setup.

\subsection{Optical Properties}

Optical properties of CPEs are determined both by backbone conformation and by backbone oxidation state. Therefore, in one hand changes in optical properties can be used to probe changes in physical state of CPEs, ${ }^{18}$ or supramolecular interaction with other molecules, as we will see in section 2.7.19 On the other hand, they can also be exploited to investigate the chemical states formed upon oxidation, as well as for the production of electrochromic devices. ${ }^{20}$

\subsubsection{Chromism}

Chromism is defined as any process leading to a color change, as a result of an alteration of the electronic states of a molecule. Different kinds of chromism are found, and can be classified depending on the stimuli leading to the color changes. Electronic states of CPEs can be modified by changes in their oxidation state or in their conjugation length. Undoped CPEs generally possess an absorption maximum a low wavelengths $(<700 \mathrm{~nm})$, generated by electronic $\pi-\pi^{*}$ transitions from the valence to the conduction band. Formation of a polaron state leads to an absorption band at higher wavelengths $(\sim 700-1100 \mathrm{~nm})$, due to the presence of mid-gap transitions involving both electrons in the valence band as well as unpaired electrons. Bipolaron state is characterized by absorption in the near-IR region, due to transitions from the valence to the bipolaron bands (Figure 2.15). 


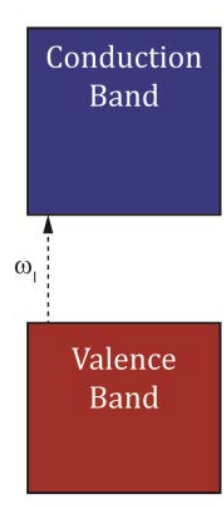

a)

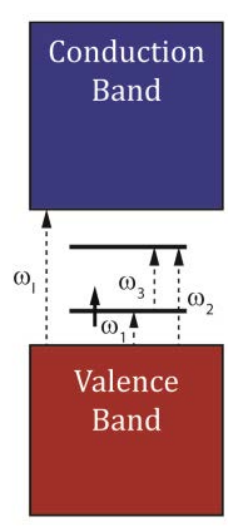

b)

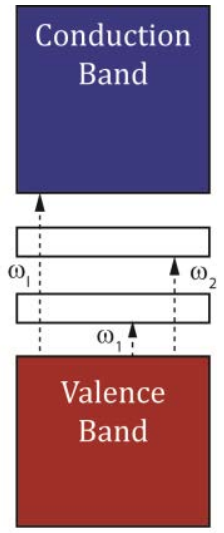

c)

Figure 2.15 Band structure of a p-type CPE, with highlighted electronic transitions: $\omega_{\mathrm{I}}=$ bandgap $\pi-\pi^{*}$ transition, and $\omega_{1-3}=$ mid-gap transitions.

As previously discussed in section 2.4, changes in $\mathbf{p H}$ determine modifications in the oxidation state of self-doped CPEs in water solution. As a result, these CPEs are halochromic. PEDOT-S in its pristine state, for example, possesses a broad absorption over all the Uv-visible and near-IR spectra, due to electronic transitions associated to a bipolaron state. Addition of $\mathrm{HClO}_{4}$ to $\mathrm{pH}=2$ does not lead to any spectral differences, as PEDOT-S is already in a highly doped state. Addition of $\mathrm{NaOH}$ to $\mathrm{pH}=14$ leads to formation of two bands at $560 \mathrm{~nm}$ and $931 \mathrm{~nm}$, associated to $\pi-\pi^{*}$ transition and polaron transitions, respectively (Figure 2.16).

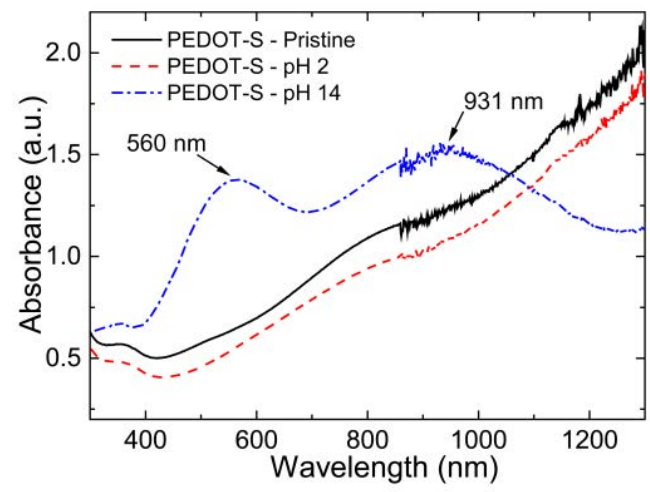

Figure 2.16 Absorption spectra of PEDOT-S in water solution $(0.1 \mathrm{~g} / \mathrm{L})$ in its pristine form (black, solid), $\mathrm{pH}=2$ (red, dashed), and $\mathrm{pH}=14$ (blue, dot-dash). 
When changes in oxidation state (and color) are triggered by an external voltage or current, the phenomena is named electrochromism. The most common technique to study electrochromic properties of CPEs is Uv-vis spectroelectrochemistry. ${ }^{21}$ With this method, changes in optical properties of the electrochromic materials in solution or in film form are monitored in situ, for application of external voltages. In Papers 1, 2 and 3 this technique has been exploited to study the chemical species formed upon oxidation in CPEs as well as CPEs blends (Figure 2.17).
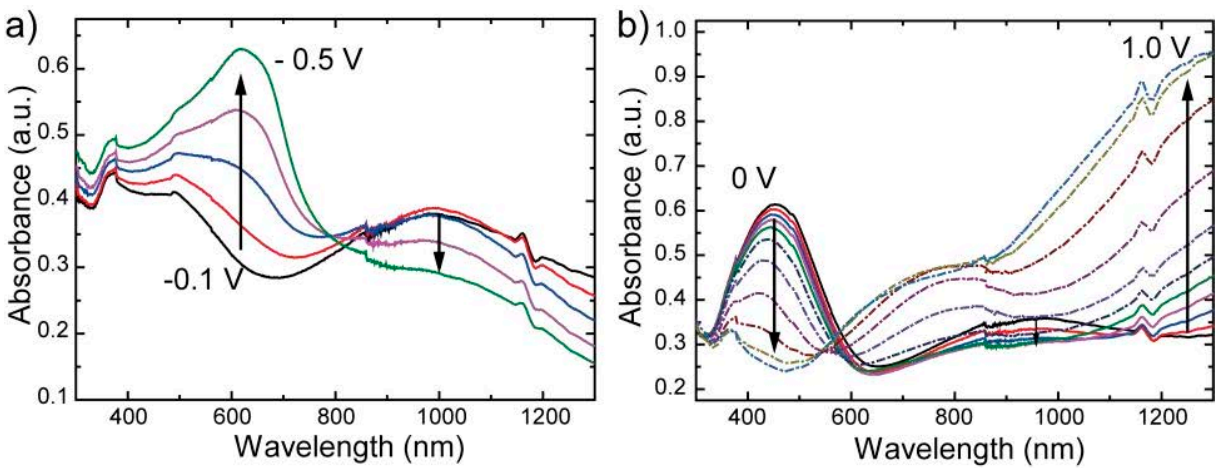

Figure 2.17 Uv-vis spectroelectrochemistry of PEDOT-S:PTEB-S 1:1 film, acquired for a) negative and $\mathrm{b}$ ) positive potentials (vs $\mathrm{Ag} / \mathrm{AgCl}$ reference electrode).

Changes in absorption spectra of the films determine variations in observed colors that can be exploited for the production of displays or electrochromic windows. For example, in Paper 1 it has been demonstrated that blending of PEDOT-S with PTEB-S leads to films exhibiting electrochromic changes within the RGB (red-green-blue) color model (Figure 2.18).

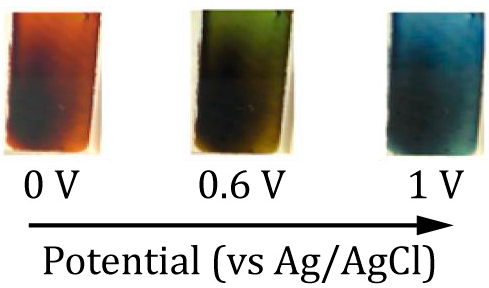

Figure 2.18 Pictures of a PTEB-S:PEDOT-S 1:1 film acquired at three different applied potentials (vs $\mathrm{Ag} / \mathrm{AgCl}$ reference electrode).

As previously mentioned, chromism can also be due to changes in CPEs conjugation length. Increase of molecular weight of CPEs generally leads to red shifts (shifts at longer wavelengths) of spectroscopic maxima. In fact, extension of the conjugation length leads to an increase of the 
number of $\pi$ electrons participating to the resonance structure. As previously described in Section 2.2, this generates a stabilization effect, and a consequent decrease of the energy difference between the ground and excited states. Instead, decrease in conjugation length results in blue shifts (shifts at shorter wavelengths) of spectroscopic maxima. CPEs conjugation length is not only determined by the chemical structure of CPEs backbone, but also by CPEs conformation. The main factors influencing CPEs conformation in solution are supramolecular interactions within CPEs chains and with the surrounding environment, as we will see in sections 2.7 and 2.8 .

\subsubsection{Emission Properties}

Photoluminescence is a physical phenomenon consisting in emission of photons from a molecule previously excited by means of light. After an electron is excited, the energy may be dissipated in a variety of ways, which are summarized by the Jablonski diagram (Figure 2.19).

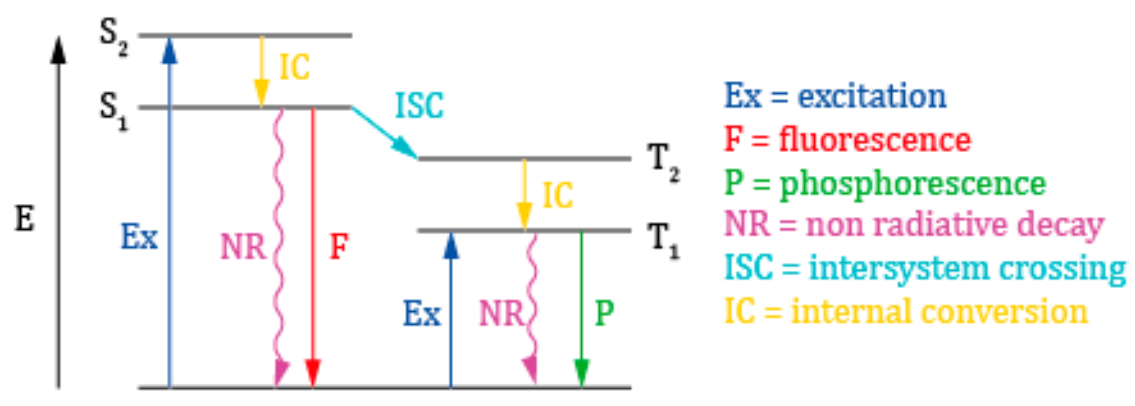

Figure 2.19 Jablonski diagram.

The word "photoluminescence" describes two different processes: phosphorescence and fluorescence. Transitions from triplet to ground state are forbidden, therefore, phosphorescence has very long lifetime (from milliseconds to seconds). As no phosphorescence has been detected for the CPEs used in this thesis, I will focus on fluorescence. Most of thiophene-based CPEs, such as PTEB-S and PTHS, exhibit emission in the Uv-vis region. However, PEDOT-S possesses intermediate bipolaron states (as previously shown in Figure 2.15), which offer non-radiative decay paths. As a result, PEDOT-S is not luminescent.

The emission spectra of CPEs are characterized by three distinctive features: the vibrational structure, the position of the emission maxima, and the intensity of the emission. The origin of the vibrational structure is described by the Frank-Condon principle, which states that during electronic transitions the nuclei are in a fixed position. As the mass 
of nuclei is higher than the one of electrons, the movement of nuclei is slow if compared to electrons. Therefore, nuclei do not have time to rearrange their position during fast electronic transitions. As a result, a straight transition occurs from the ground $S_{0}$ to the excited $S_{1}$ state. If the nuclear distances of the ground and excited states are similar, the most probable transition is the $v=0 \rightarrow v^{\prime}=0$, and transitions to higher vibrational states are less probable. If nuclear distances of ground and excited states are different, higher vibrational states are excited during the electronic transition (Figure 2.20). Differences in intensity of the observed vibrational peaks are due to different probabilities of the transitions to occur.

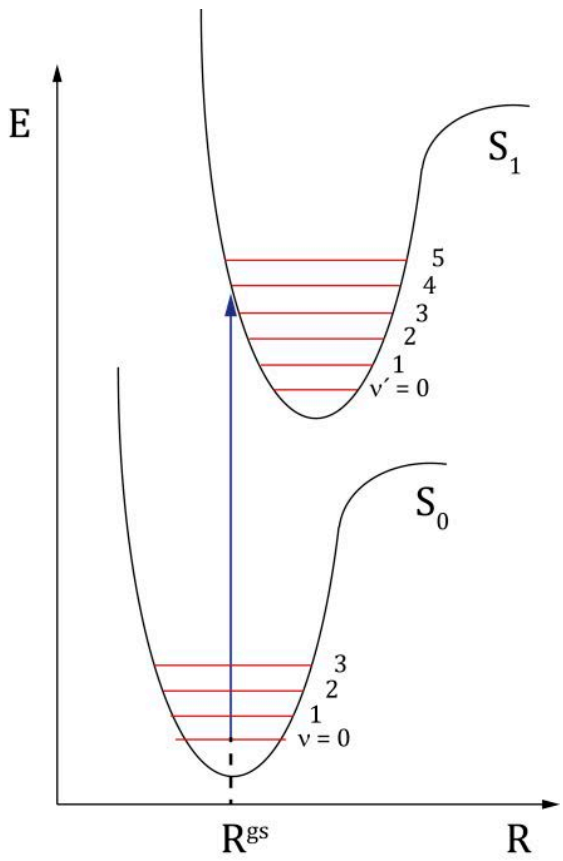

Figure 2.20 Profile of the bands of the ground $\left(\mathrm{S}_{0}\right)$ and excited $\left(\mathrm{S}_{1}\right)$ states. $\mathrm{E}$ is the energy, $\mathrm{R}$ is the displacement coordinate, $\mathrm{Rgs}$ is the coordinate of the nuclei in the ground state.

The positions of the emission maxima are determined by CPEs conjugation length, as described in section 2.6.1. As for absorption spectra, emission maxima are defined by the bandgap energy. A third factor of interest is the emission intensity. Quenching is defined as any phenomena that decrease the emission intensity of a substance. This process can be classified as dynamic or static depending on its nature. Dynamic quenching is due to collision between molecules, while static 
quenching occurs when the fluorophore and the quencher form a complex in the ground state.

\subsection{Self-assembly of CPEs}

As defined in section 2.3, CPEs are constituted by a hydrophobic backbone and hydrophilic side groups and are, consequently, amphiphilic molecules. The conjugated backbones are, therefore, soluble in non-polar solvents and can undergo supramolecular interactions such as $\boldsymbol{\pi}$ - $\boldsymbol{\pi}$ stacking. In contrast, the side groups provide solubility in polar solvents. Their charges give the possibility of attractive electrostatic interactions with oppositely charged species, as well as repulsion with species possessing the same charge. The electronegativity of atoms composing the side groups leads to the possibility of forming hydrogen bonds with polar protic molecules, such as water. As a consequence, it is not straightforward to predict behaviors of CPEs such as solubility or formation of supramolecular assemblies. Early studies of CPEs behavior in solution lead to the conclusion that CPEs dissolution in polar solvents, such as water, does not leads to simple solutions of isolated chains, but rather to CPEs aggregates surrounded by solvent molecules. ${ }^{22}$

\subsubsection{Aggregation of CPEs in Solution}

Due to their amphiphilic nature, when several CPE molecules are dissolved in polar solvents, they have the tendency to form aggregates. These aggregates are supramolecular structures where the hydrophobic backbones are shielded from the surrounding solvent, while the charged groups are exposed to the solvent molecules. The shape and dimensions of these aggregates depend on several factors, such as backbone structure, nature and length of the side chains, atoms composing the side groups, and on the type of counterions. As anticipated in section 2.6, changes in absorption and emission spectra can provide useful information on the aggregation state of CPEs. In Paper 3 it has been shown that PTHS conformation in solution can be tuned by dissolution in solvents having different polarity (such as water and methanol). ${ }^{23}$ When PTHS is dissolved in polar solvents, such as water, red shifted emission and absorption maxima are observed with respect to less polar solvents, such as methanol (Figure 2.21). This is due to stronger aggregation of hydrophobic PTHS backbones in highly polar solvents. Formation of aggregates in water also leads to inter-chain quenching and decrease of observed emission intensity. 

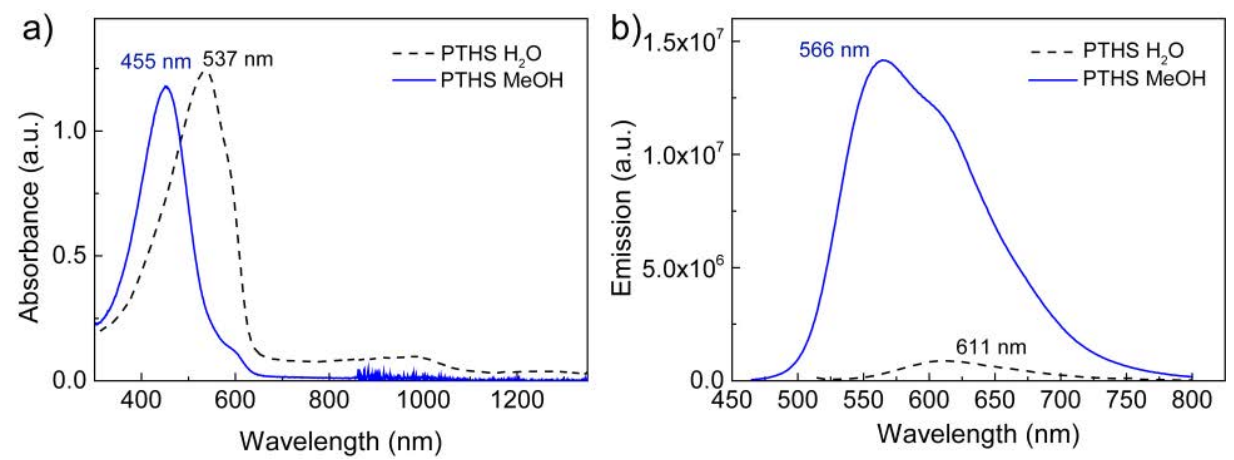

Figure 2.21 a) Absorption and b) emission spectra of PTHS in water (black, dash) and methanol (blue, solid). PTHS concentration was $0.0613 \mathrm{~g} / \mathrm{L}$ for absorption and 0.00613 $\mathrm{g} / \mathrm{L}$ for emission.

Variation of optical properties as function of chosen solvent is named solvatochromism. The aggregation behavior of CPEs in solution can be tuned by different means such as addition of additives in form of small molecules, co-solvents or surfactants, ${ }^{24}$ or by blending with other CPEs. ${ }^{22}$

\subsubsection{Tuning CPEs Solubility by Counterion Exchange}

As mentioned in previous section, different counterions can affect conformation and aggregation of polymer chains. In particular, bulky counterions inhibit interactions among CPEs chains and prevent aggregates formation. ${ }^{25}$ In Papers 2, 3, and 5 it has been shown that the hydrophilic/hydrophobic character of CPEs or CPEs blends can be modified by counterion exchange with positively charged surfactants. Electrostatic interaction of a self-doped CPE (PEDOT-S) with certain ammonium salts leads to formation of PEDOT-S:ammonium salts. The produced complexes are the result of the electrostatic interaction between the negatively charged sulfonate groups of PEDOT-S and the positively charged ammonium groups of the surfactants (such as dioctylammonium, $(\mathrm{Oct})_{2} \mathrm{NH}_{2}{ }^{+}$), leading to the following equilibria:

$$
\text { PEDOT-S- } \mathrm{H}^{+}+(\mathrm{Oct})_{2} \mathrm{NH}_{2}{ }^{+} \mathrm{Cl}^{-} \rightleftharpoons \text { PEDOT-S }:(\mathrm{Oct}){ }_{2} \mathrm{NH}_{2}{ }^{+} \downarrow+\mathrm{HCl}
$$

The key point of this strategy is the PEDOT-S:ammonium hydrophobicity, which leads to product precipitation from water (Figure 2.22). The water-soluble excess of ammonium salts can be easily removed by centrifugation/washing cycles. The dried complexes can then be redissolved in organic solvents such as chloroform or chloroform:methanol. 


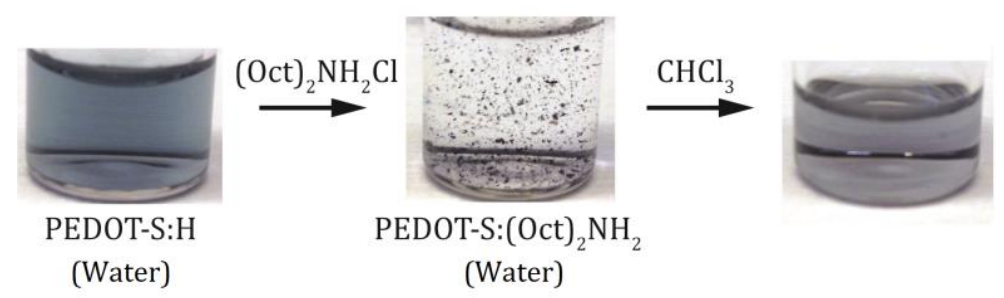

Figure 2.22 Schematic representation of three major steps leading to PEDOT-S:(Oct) ${ }_{2} \mathrm{NH}_{2}$ preparation and dissolution in organic solvents.

In Paper 4 the concept has been extended to another self-doped CPE (PTHS) and to PTHS:PEDOT-S blends. PTHS interaction with dioctylammonium chloride results in the formation of a water-soluble complex. Therefore, a more hydrophobic ammonium salt (trioctylammonium chloride) has been chosen, which leads to complex precipitation. Blending PTHS and PEDOT-S from water solutions leads, instead, to complex precipitation in presence of $(\mathrm{Oct})_{2} \mathrm{NH}_{2} \mathrm{Cl}$. Dissolution of [PTHS:PEDOT-S(1:1)]:(Oct ${ }_{2} \mathrm{NH}_{2}$ in chloroform leads to a solution possessing very different optical properties with respect to PTHS:PEDOT$S$ blend in water, which can be attributed to a different conformation of CPEs chains (Figure 2.23).

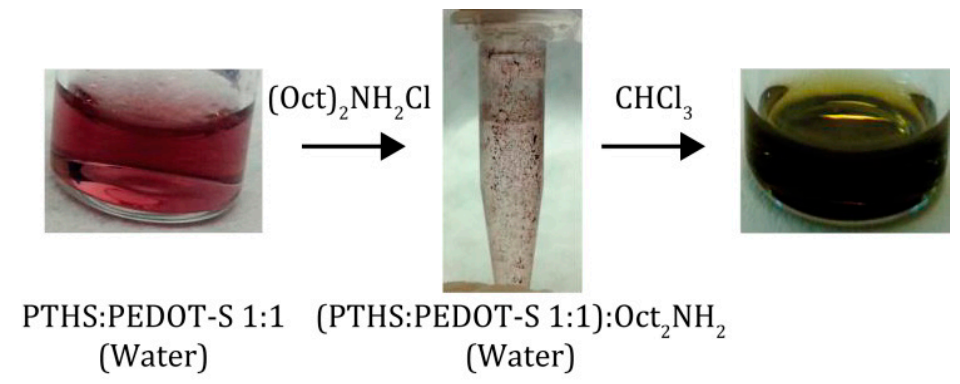

Figure 2.23 Schematic representation of the complexation process of PTHS:PEDOT-S blend 1:1 with dioctylammonium chloride and consequent complex dissolution in chloroform.

\subsubsection{Blending}

CPs solubility in common solvents enables the preparation of composite materials by simply mixing solutions of the single components. Previous observations on the behavior of conjugated polymers blends have revealed that phase separation can occur when two different CPs are mixed together. The reason is a low dissolution entropy of the CPs chains 
if compared to small molecules, which hinders CPs mixing. ${ }^{26}$ When polymer blends are casted to form a thin film, phase separation can lead to macroscopic features that decrease devices efficiency, due to poor contact between the CP phases. Control of phase separation in polymer blends is, therefore, essential for production of homogeneous films for high performance devices. ${ }^{27}$ In paper 1 it has been demonstrated that blending two CPEs (PTEB-S and PEDOT-S) from aqueous solutions can be used as a strategy to produce homogeneous CPEs mixtures. The blends have been consequently used to produce films having enhanced electrical and optical properties with respects to films from pure polyelectrolytes (Figure 2.24).

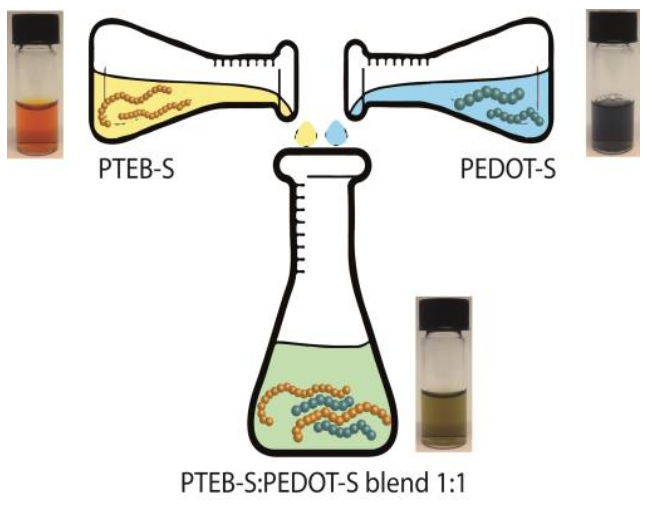

Figure 2.24 Schematic representation of blending PTEB-S and PEDOT-S from water solution to form a PTEB-S:PEDOT-S blend 1:1.

In Paper 3 the concept of blending has been combined with the one of "counterion exchange" (explained in section 2.7.2), in order to produce new PTHS:PEDOT-S blends with the desired properties. The blends casted from water or methanol have been exploited for the production of films for organic electrochemical transistor devices, as it will be discussed in chapter 3.

\subsection{Film Formation}

Device fabrication requires the deposition of CPEs to form a thin film. Various deposition techniques are available, depending on the desired characteristics of the films, as well as thicknesses and homogeneity. In this thesis, solution processing techniques have been used to form CPEs thin films. For electrochromic characterisation of the films, where high 
film thickness (in the micrometres range) is of importance to evaluate the absorption signature, drop casting has been used. This technique involves the deposition of CPEs solution on a substrate to produce a film. However, drop casting is not the best technique for devices production, as the produced films does not have homogeneous thicknesses. Spin coating has therefore been used to generate homogeneous films for devices fabrication. When solution processing techniques are used, the properties of the formed films strictly depend on the aggregation state of the species in solution. Therefore, as mentioned in section 2.7.3, control of aggregation of the CPEs in solution is of primary importance in order to produce films having desired properties. In order to study films formation and morphology, two techniques have been used in this thesis: spectroscopy and atomic force microscopy (AFM).

\subsubsection{Drying Films Characterization}

The deposition process can be followed by monitoring the emission spectra of the components while films are drying from wet to solid state. Decrease in emission intensity is generally observed during the drying process, due to inter-chain quenching as the solvent evaporates and the polymer chains come in contact with each other. Shifts in emission maxima and/or appearance of vibronic fine structures can give useful information on the microstructure formation of the films. In Paper 1 this method has been used to appreciate differences in microstructures of PTEB-S and PTEB-S:PEDOT-S films. PTEB-S deposition from solution to wet films leads to a red shift in emission maxima. This can be attributed to $\pi-\pi$ stacking of the conjugated PTEB-S backbones in the wet film. Films drying did not lead to any changes in emission maxima, meaning that PTEB-S conformation is unchanged from wet to solid state. A blue shift in blend emission has been observed for PTEB-S:PEDOT-S films, attributed to the formation of mixed aggregates during the drying process (Figure 2.25). 

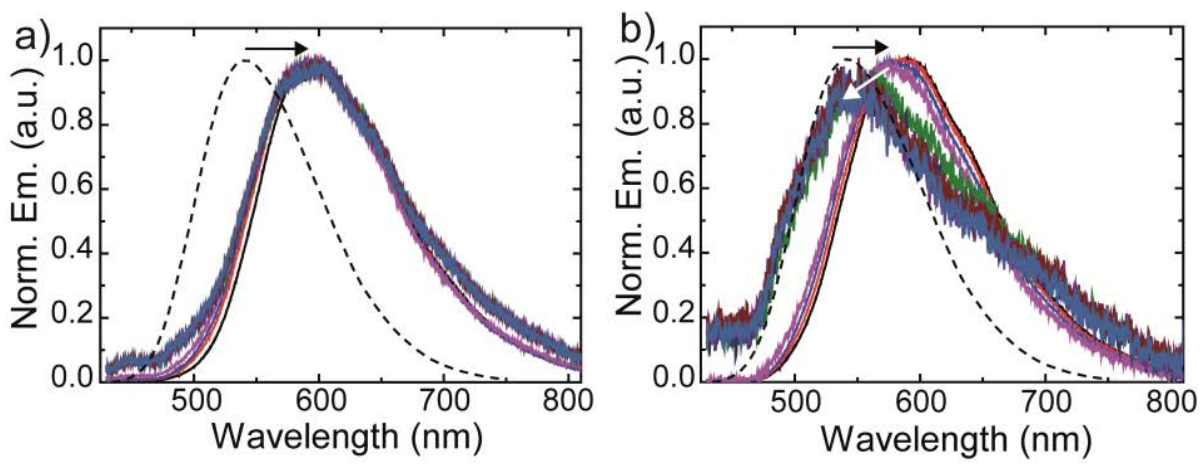

Figure 2.25 Normalized emission spectra of a) PTEB-S and b) PTEB-S:PEDOT-S 1:1 films while drying.

\subsubsection{Film Characterization}

Spectroscopic methods have been used to investigate the properties of the formed films. Absorption and emission spectroscopies have been used in Papers 1, 3 and 4 to monitor the optical properties of the formed films. In Paper 3, for example, it has been shown that changes of the PEDOT-S counterion lead to PTHS:PEDOT-S blends, where cast films from water or methanol have different spectroscopic properties (Figure 2.26). In particular, a red shift has been observed in PTHS:PEDOT-S films cast from water, which can be related to the increase of PTHS conjugation length in aggregate form. Emission data shows, instead, a blue shift and quenching of PTHS:PEDOT-S films, if compared with PTHS:[PEDOT-S:(Oct $)_{2} \mathrm{NH}_{2}$ ] films cast from methanol. The difference could be related to higher counterion steric hindrance in PTHS:[PEDOT-S:(Oct) ${ }_{2} \mathrm{NH}_{2}$ ] blend, which causes an increase in distance between CPEs backbones. 

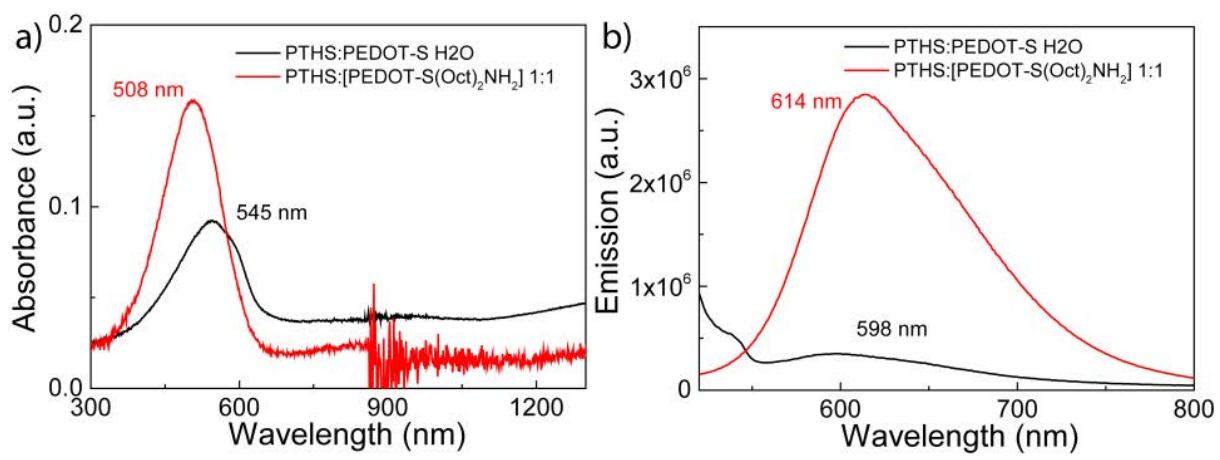

Figure 2.26 a) Absorption and b) emission data of spin coated films of PTHS:PEDOT-S 1:1 (black, $8.14 \mathrm{~g} / \mathrm{L}$ ) from water solution, and PTHS:[PEDOT-S:(Oct) ${ }_{2} \mathrm{NH}_{2}$ ] 1:1 (red, $1 \mathrm{~g} / \mathrm{L}$ ) from methanol. Excitation wavelength was $450 \mathrm{~nm}$.

Electron spin resonance spectroscopy (ESR) has been exploited in Paper 1 for the characterization of the polaron states formed in PEDOT-S and PTEB-S:PEDOT-S films upon PEDOT-S oxidation. As mentioned in section 2.2, PEDOT-S is found in a polaron state, possessing an unpaired electron. PTEB-S, instead, show no ESR signal, as it is directly oxidized from a neutral to a bipolaron state. In ESR, the differences in resonance energies observed for different molecules are expressed by variations of g-factor, which is a parameter characteristic of the molecule where is located the unpaired electron. For PEDOT-S, the g-factor is very close to 2 , which is the value observed for organic radicals (Figure 2.27, a). Moreover, g-factor anisotropy has been recorded for different positions of PEDOT-S films (parallel or perpendicular with respect to the applied magnetic field), which can be attributed to the preferential orientation of PEDOT-S chains in a film form.

Moreover, ex situ ESR spectroelectrochemistry has been used to record differences in signal magnitude as function of the applied voltage (Figure $2.27, \mathrm{~b})$. 

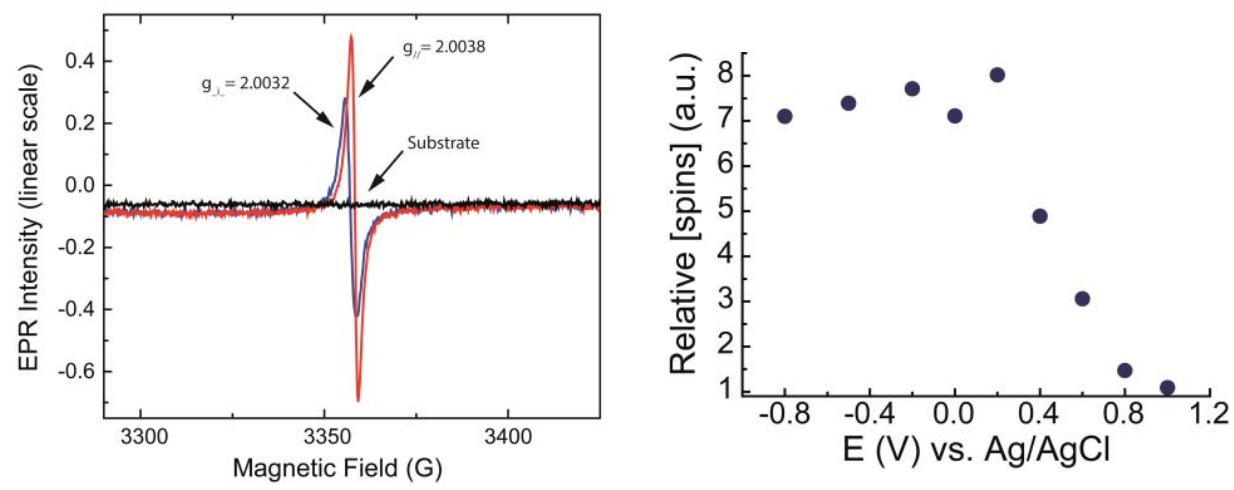

Figure 2.27 ESR data of a) PEDOT-S film positioned parallel (red) or perpendicular (blue) to the magnetic field and b) PEDOT-S film as function of the applied potential (vs $\mathrm{Ag} / \mathrm{AgCl}$ reference electrode).

Atomic force microscopy (AFM) has been exploited in papers 1, 3 and 5 to study the morphology of formed films. The data show interesting differences in film morphology upon blending. Fibril-like structures have been observed for films of the blends, which are probably due to mixed aggregates formation (Figure 2.28). These changes have been related to different ion transport ability of the CPEs films and differences in device characteristics, as we will see in the next chapter.
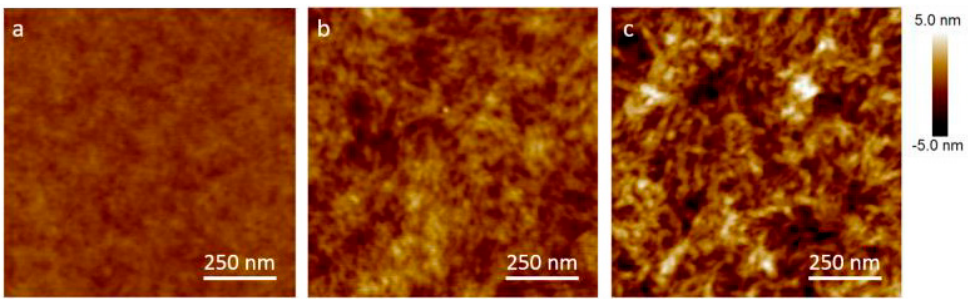

Figure 2.28 AFM topography images of a) PTEB-S, b) PEDOT-S, and c) PTEB-S:PEDOT-S 1:1 spin-coated films.

Moreover, in Paper 6 AFM measurements have been performed on films of spin-coated phospholipids both in air and water, in order to assess structural differences connected to biomembranes formation from dried to wet state. ${ }^{28}$ 


\section{Chapter 3}

\section{Electrochemical Transistors}

Transistors are electrical devices capable of signal amplification and/or transduction. Different types of transistors can be fabricated, and they are classified depending on the nature of their components as well as their working mechanism. This work is focused on a particular class of transistors called organic electrochemical transistors (OECTs). As we will see, these are three terminal devices that comprise a conjugated polymer (CP) or conjugated polyelectrolyte (CPE), and an electrolyte as devices components.

Due to easy fabrication and CPs processability, OECTs found application in printed electronics, for the production of displays and biosensors. ${ }^{29-32}$ Additionally, low operating voltages, stability in aqueous environment, and capability of interfacing ionic and electronic signals make them suitable devices for bioelectronics and healthcare applications. ${ }^{33,34}$ However, despite their great success, OECTs currently suffer of many drawbacks, which limit their use for practical applications. The main challenges regard increase of active materials redox and water stability for prolonged devices operation as well as for the use of water-based electrolytes, which is a requirement to interface with biological systems. Moreover, decrease of switching times, and increase of signal amplification are also desired.

\subsection{OECTs Building Blocks}

OECTs are made of three terminals, named the drain, the source and the gate. The drain and the source are connected to each other by a redox active specie, called the active material. All the OECTs produced so far have CPs or CPEs as active material components. The active material is then connected to the gate electrode by means of an electrolyte (Figure 3.1). 


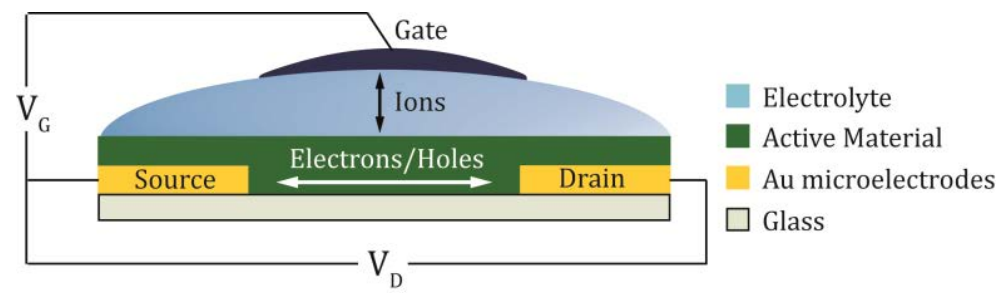

Figure 3.1 Schematic representation of an organic electrochemical transistor device.

In order for the device to work, a drain voltage $\left(V_{D}\right)$ is applied between source and drain to enable the flow of charge carriers (electrons or holes) through the channel. The gate modulates the redox state of the active material by injecting or extracting ions from/to the electrolyte and, therefore, shifting active material redox equilibria toward the conductive $(\mathrm{ON})$ or non-conductive (OFF) form. OECTs function depends both on the efficiency of the active material to transport electrical charges, and on the ability of the gate to supply the channel with ions and, thereafter, to modulate the equilibria of the redox active specie. The latter feature hinges on the area and on the kind of material composing the gate electrode, ${ }^{35,36}$ and on the mobility of the ions composing the electrolyte. ${ }^{37}$ Another factor influencing devices performance is the morphology of the active material. ${ }^{38}$ Changes in films morphology affect both the electronic and the ionic transport ability of the CPs constituting the active material. Therefore, understanding the relation between the state of the materials in solution and films morphology, as shown in section 2.8, is the key to produce materials having the desired properties and leading to devices possessing the desired performance.

\subsection{OECTs Characterization}

Mainly three kinds of measurements are required in order to characterize transistor devices (Figure 3.2). ${ }^{39}$ Steady-state, output characteristics are acquired by measuring the drain current $\left(I_{D}\right)$ for drain voltage $\left(V_{D}\right)$ sweeps, at fixed gate bias $\left(\mathrm{V}_{\mathrm{G}}\right)$. Negative drain voltages have been applied for all OECTs characterization. In fact, as previously discussed in section 2.4, all the produced devices comprise p-type CPEs as active material, whose main charge carriers are holes. Application of positive drain voltages would also be possible, but would require higher gate voltages to switch OFF the devices. ${ }^{39,40}$ Output curves delineates the limits between linear and saturated regimes of devices operation, and allow selection of the lowest drain voltages necessary to have best device performances without compromise active materials redox stability. Transfer 
characteristics are, instead, acquired by measuring the $I_{D}$ at constant $V_{D}$, for $V_{G}$ sweeps. Shape and hysteresis of the curves are useful to understand transient processes such as ions insertion/ejection or overcharging effects in the active material. ${ }^{41}$ The first derivative of the transfer curve leads to a parameter called transconductance $\left(\mathrm{g}_{\mathrm{m}}=\right.$ $\left.\delta \mathrm{I}_{\mathrm{D}} / \delta \mathrm{V}_{\mathrm{G}}\right)$, which is a measure of devices amplification. The best transconductance value reported so far is $20 \mathrm{mS} / \mathrm{cm}$ for drop casted semiconducting materials. ${ }^{42}$

Transient responses are measured by recording the drain current for gate voltage switches at fixed drain voltage. This measurements allow to appreciate the switching ability of the devices and the amount of time required to oxidize and reduce the active material, and therefore switch $\mathrm{ON}$ and OFF the devices, respectively.
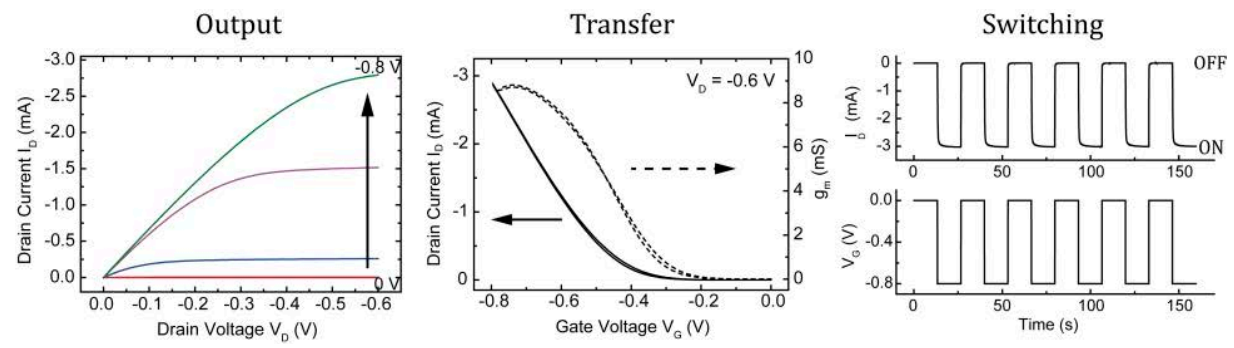

Figure 3.2 Output, transfer and switching characteristics acquired for devices having PTHS as active material.

\subsection{Operation Modes of OECTs}

OECTs can mainly operate in two modes, depending on the nature of the active material: depletion or accumulation mode. In depletion mode devices the active material is in a doped, highly conductive state in its pristine form, and the device is $\mathrm{ON}$ at zero gate bias (Figure 3.3). Application of positive gate biases drives positive ions from the electrolyte into the active material, and switch OFF the device. Devices having PEDOT:PSS or PEDOT-S as active material work in depletion mode, according to the follow equilibria:

$$
\mathrm{PEDOT}^{+} \mathrm{S}^{-}+\mathrm{M}^{+}+\mathrm{e}^{-} \rightleftharpoons \text { PEDOT }^{0} \mathrm{~S}^{-}: \mathrm{M}^{+}
$$




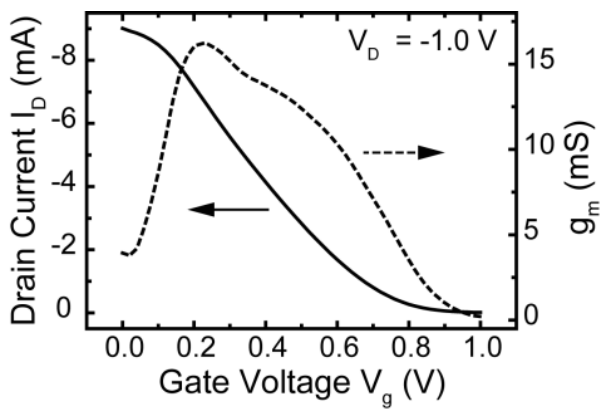

Figure 3.3 Transfer characteristics (solid) and related transconductance (dashed) of PEDOT-S OECTs.

Currently, most of the produced devices operate in depletion mode. The main reason is that most of devices produced so far comprise PEDOT:PSS as active material, due to its superior features in terms of stability and conductivity.

In accumulation mode devices, the active material is in a neutral, nonconductive state and the device is OFF at zero gate bias (Figure 3.4). Application of negative gate biases leads to either positive ions extraction or negative ions insertion into the active material, and switch $\mathrm{ON}$ the device. PTEB-S and PTHS devices work in accumulation mode according to the following equilibria:

$$
\begin{aligned}
& \text { PTEB }^{0} S: \mathrm{Na}^{+} \rightleftharpoons \mathrm{PTEB}^{+} \mathrm{S}^{-}+\mathrm{Na}^{+}+\mathrm{e}^{-} \text {, } \\
& \mathrm{PTEB}^{0} \mathrm{~S}^{-}: \mathrm{Na}^{+}+\mathrm{X}^{-} \rightleftharpoons \mathrm{PTEB}^{+} \mathrm{S}^{-}+\mathrm{Na}^{+}: \mathrm{X}^{-}+\mathrm{e}^{-} \\
& \text {and }
\end{aligned}
$$

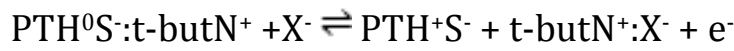

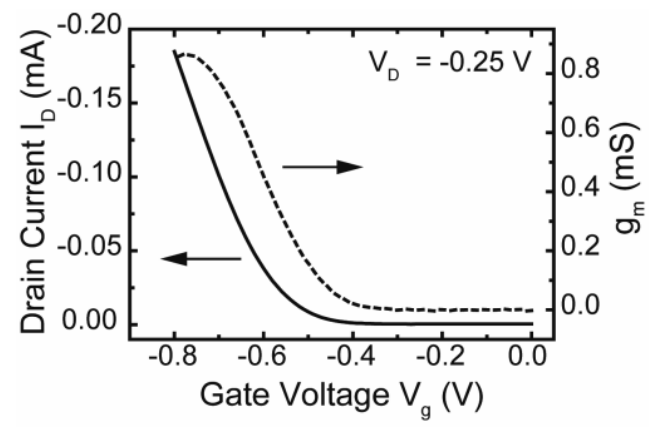

Figure 3.4 Transfer characteristics (solid) and related transconductance (dashed) of PTEB-S OECTs. 
The main advantage of accumulation mode devices is their low power consumption, as they are normally switched OFF, and can be switched ON on-demand. These kinds of OECTs are particularly interesting for sensing applications, where a "switching ON" event is more easily detectable than a "switching OFF" one. However, most of the produced devices could not find practical applications, as they could not meet the high standards imposed by depletion mode devices. Only recently, synthetic efforts have been spent on the development of new semiconducting materials with improved characteristics for the production of better accumulation mode OECTs. ${ }^{38,42}$

\subsubsection{Tuning OECTs Operation Mode}

Fine tuning of devices operation can be achieved by careful choice of the gate material or by modifying the channel geometry. ${ }^{43}$ In Paper 1 , it has been demonstrated that, with similar gate material and channel geometry, OECTs operation mode can also be tuned by blending materials having different properties. In particular, blending two CPEs having metallic and semiconducting behavior has been used as an approach to produce devices showing a hybrid accumulation/depletion operation mode (Figure 3.5). PEDOT-S and PTEB-S have been mixed together from water solutions, in presence of a surfactant, to form CPEs blends (as shown in section 2.7.3). Films of the blends have shown enhanced characteristics with respect to films made from single components, such as enhanced electrochromic properties, as previously demonstrated in section 2.6.1.

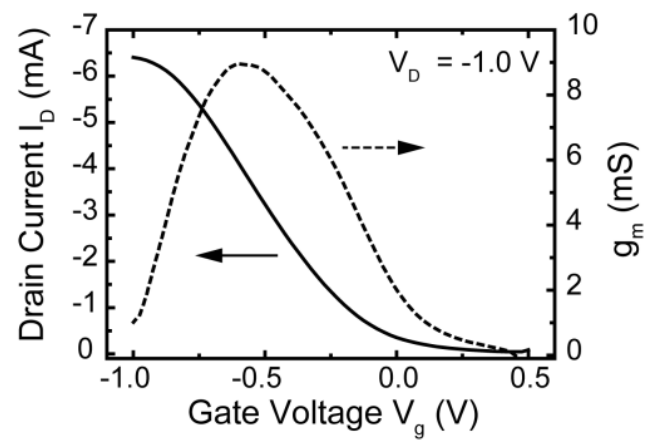

Figure 3.5 Transfer characteristics (solid) and related transconductance (dashed) of PTEB-S:PEDOT-S 3:2 OECTs.

The operation mode of devices could be tuned by modification of blend stoichiometry. OECTs showed also improved characteristics with 
respect to devices made by single components, possessing better stability as well as lower switching times, as we will see in the next section.

In Paper 2 PEDOT-S devices have been fabricated, however, the choice of the gate electrode (Ag/AgCl instead of PEDOT:PSS) influenced the operation mode of the devices, which now operate in an hybrid accumulation/depletion mode. Moreover, the threshold voltage of OECTs could also be tuned by counterion exchange with certain ammonium salts, as shown in section 2.7.2, demonstrating that also CPEs counterions play a role in devices operation (Figure 3.6).

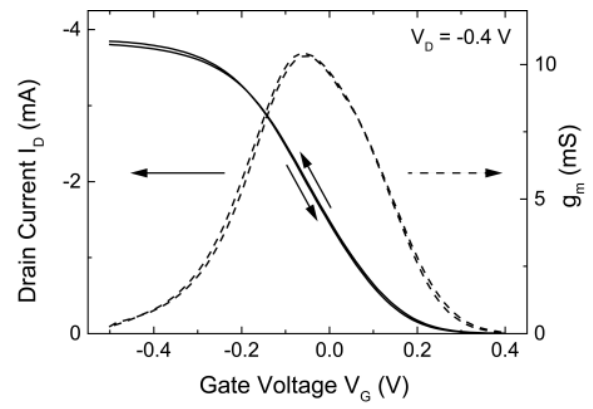

Figure 3.6 Transfer characteristics (solid) and related transconductance (dashed) of PEDOT-S:(Oct) ${ }_{2} \mathrm{NH}_{2}$ OECTs.

The two above described concepts have been combined in Paper 3, where blending PEDOT-S with PTHS has been accomplished from water or methanol, depending on the nature of PEDOT-S counterion. Accumulation mode devices have been produced, operating at different gate voltages and exhibiting enhanced properties with respect of devices made with single components (Figure 3.7).
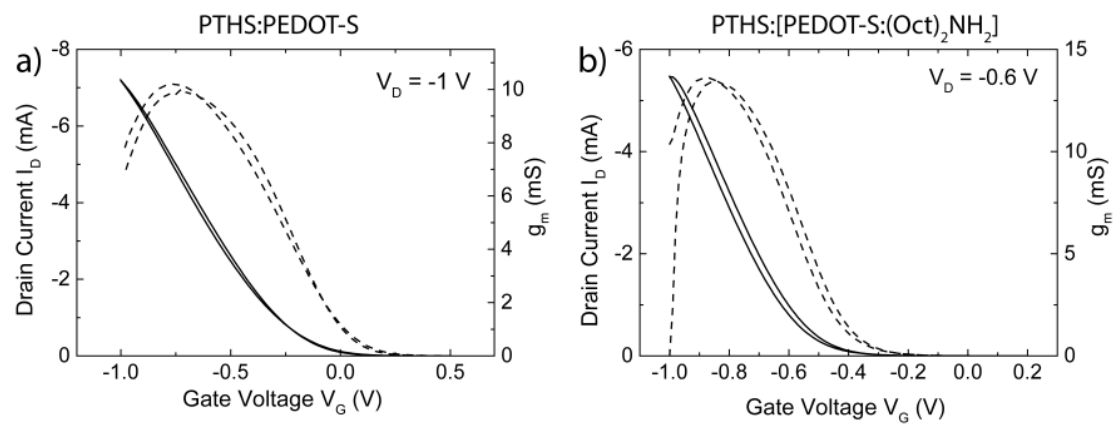

Figure 3.7 Transfer characteristics (solid) and related transconductance (dashed) of a) PTHS:PEDOT-S 1:1 and b) PTHS:[PEDOT-S:(Oct) ${ }_{2} \mathrm{NH}_{2}$ ] 1:1 OECTs. 


\subsection{Switching Times in OECTs}

Fast switching is a desirable property for sensors, logic circuits and displays applications. ${ }^{29,44}$ Therefore, a great effort has been spent on improve OECTs switching times. ${ }^{45}$ In this work time constants for $\mathrm{ON}$ and OFF processes have been defined as the time required for reach the $90 \%$ $\left(\tau_{\mathrm{ON}}\right)$ and the $10 \%\left(\tau_{\mathrm{OFF}}\right)$ of the maximum currents, respectively. ${ }^{46}$ Mobility of the charge carriers within the active material combined with insertion/ejection processes of ions from the electrolyte, as well as the length of transport are the rate determining step for switch ON and OFF of devices. The mechanism of ions diffusion from/to the active material is still a matter of debate, and can be influenced by several factors. As discussed in the previous section, film morphology plays an important role, as it determines the interface between the redox active species and the ionic ones from the electrolyte. In previous reports, it has been observed that changes in morphology upon additive addition has an effect on ion transport of the films, and consequently on devices performance. ${ }^{38}$ In paper 1 blending PEDOT-S and PTEB-S leads to devices having shorter switching times with respect to devices made by pure components (Figure 3.8).
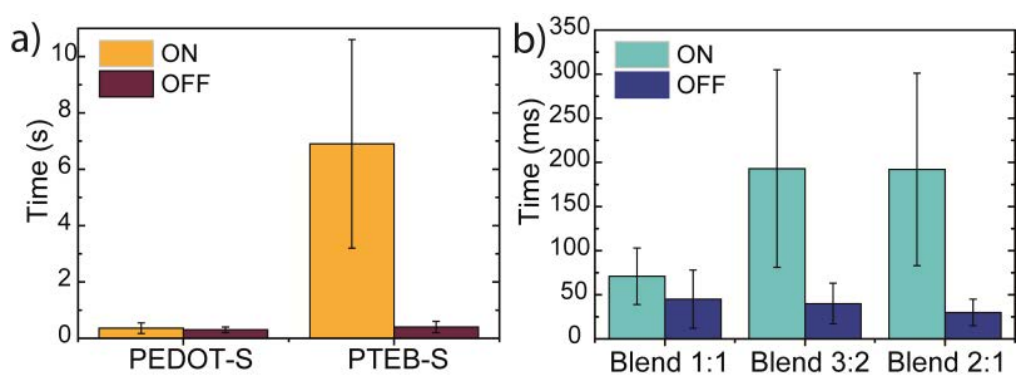

Figure 3.8 Switching times for a) OECTs made of pure PEDOT-S and PTEB-S components and b) OECTs having PTEB-S:PEDOT-S blends as active materials.

The effect has been attributed to improved ion transport of the blends, which could be related to changes in morphology of the films. In fact, films made of pure components show very homogeneous features, while a distinct fibrillar morphology could be observed for films of the blends, as shown in section 2.8.2. Similar results on switching times have been observed in Paper 3.

In Paper 2 it has been demonstrated that the chosen electrolyte has a great impact on OECTs switching times. Devices made by the same active material (PEDOT-S:(Oct) ${ }_{2} \mathrm{NH}_{2}$ ) and possessing same $\mathrm{Ag} / \mathrm{AgCl}$ gate and same channels geometry exhibit very different switching times when 1,1- 
butyl-3-methylimidazolium tetrafluoroborate $\left(\mathrm{BMIMBF}_{4}\right)$ ionic liquid or 1 $\mathrm{M} \mathrm{KCl}$ in water are used as electrolytes (Figure 3.9). Differences in ions mobility and dimensions are likely to play a role in switching processes. However, further investigations would be needed to evaluate the importance of the nature of electrolyte ions in gate/electrolyte and electrolyte/polymer processes.

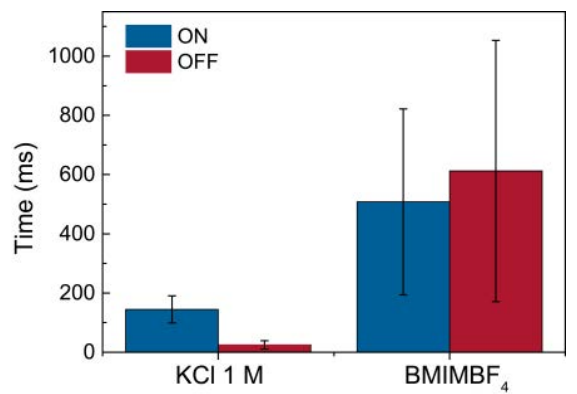

Figure 3.9 Switching times for PEDOT-S:(Oct) ${ }_{2} \mathrm{NH}_{2}$ devices comprising $1 \mathrm{M} \mathrm{KCl}$ in water or $\mathrm{BMIMBF}_{4}$ ionic liquid as electrolytes.

\subsection{OECTs Stability}

The properties of the active material have an impact on devices stability. The redox stability of the electroactive specie is of importance for long time operation of the devices, while its physical-chemical properties define which electrolyte can be used for devices fabrication. For example, if the active material is a water-soluble specie, a non-aqueous electrolyte should be used to prevent film dissolution. The properties of the electrolyte and the active material should also match the specific application. Water-based electrolytes are used for printed electronics, but are not a requirement. On the other hand, interface electronics devices with the biological world necessitates the use of water-based electrolytes, due to biological systems compatibility.

\subsubsection{Stability towards Cycling}

An important limiting factor for the use of OECTs in practical applications is their poor stability upon cycling, indicated by a decrease of the observed drain currents. Degradation processes can be attributed to several causes. Poor redox stability of the active material can lead to its degradation by oxidative processes. ${ }^{47}$ Non reversible insertion/ejection of ions from/to the active material can affect the 
switching processes, and can be related to ions trapping into the polymeric matrix as well as poor morphology of the active material. To avoid active material degradation it is important to choose polymeric materials that show high redox stability and that can be oxidized at very low voltages. ${ }^{48}$ The nature of the conjugated backbone is a key factor for CPs redox stability. ${ }^{49}$ Conjugated polymers based on EDOT monomers have shown very good redox stability and low oxidation potentials, and currently represent the most used materials for OECTs production. In Paper 1 it has been demonstrated that blending a conjugated polyelectrolyte (PTEB-S) having low redox stability with a highly stable one (PEDOT-S) has a positive effect in devices stability upon switching (Figure 3.10).
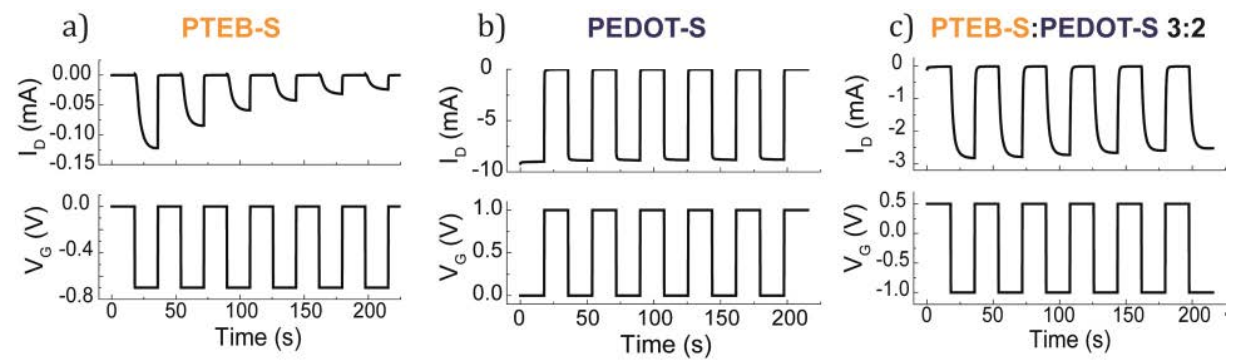

Figure 3.10 Switching characteristics of a) PTEB-S, b)PEDOT-S, and c) PTEB-S:PEDOT-S $3: 2$ based devices.

The ionic species constituting the electrolyte are also very important to produce devices that can be operated for long time without currents loss. Comparison of PEDOT-S:(Oct) ${ }_{2} \mathrm{NH}_{2}$ devices having $\mathrm{KCl}$ in water or ionic liquid as electrolytes (Paper 2) clearly showed that choice of the electrolyte have a great impact on devices stability towards cycling (Figure 3.11). This can be related to trapping of ions into the active material, leading to both a diminution of maximum drain currents and an increase of the OFF currents, due to a decrease of device switching speed with time. 

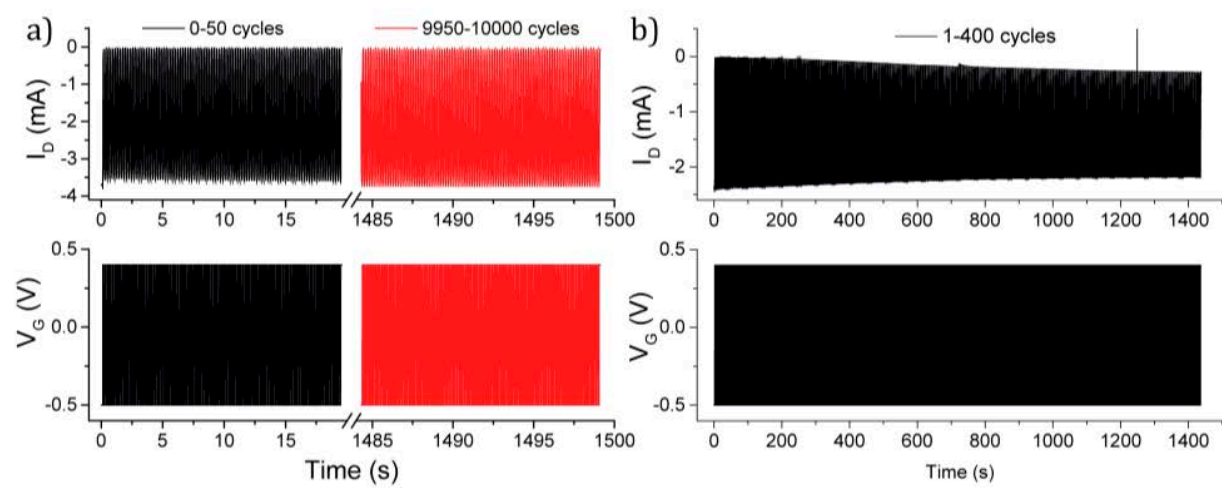

Figure 3.11 Stability measurements upon switching for PEDOT-S:(Oct) ${ }_{2} \mathrm{NH}_{2}$ devices comprising a) $1 \mathrm{M} \mathrm{KCl}$, and b) $\mathrm{BMIMBF}_{4}$ ionic liquid as electrolytes.

Therefore, use of electrolytes comprising small ions will be desirable for future development of stable devices. For this reason, it is important to tune the properties of the active materials to render them compatible with water-based electrolytes.

\subsubsection{Stability in Water-based Electrolytes}

CPEs possess high water solubility due to their ionic functionalities. Moreover, addition of dopants, like in the case of the polymeric dispersion PEDOT:PSS, leads to materials having pronounced water solubility, as discussed in section 2.3. As a consequence, fabrication of OECTs comprising a water-based electrolyte is not possible due to films degradation. Additives and cross-linking agents have been extensively employed to promote film adhesion and prevent dissolution. However, these components are typically insulating and generally lead either to a decrease of electronic properties of the active material or changes in morphology affecting ion processes. ${ }^{50,51}$ Change of the fabrication strategies and the substrate resulted in additive free PEDOT:PSS films exhibiting good stability in water. ${ }^{52}$ Hydrophobic semiconducting materials could also be used to produce accumulation mode OECTs. ${ }^{42}$ In Paper 2 it has been shown that hydrophobic PEDOT-S:ammonium salts can be used to prepare films exhibiting high stability in water solution. The hydrophobic CPEs could, therefore, be incorporated as additive-free active materials for OECTs comprising a water-based electrolyte (Figure 3.12). 


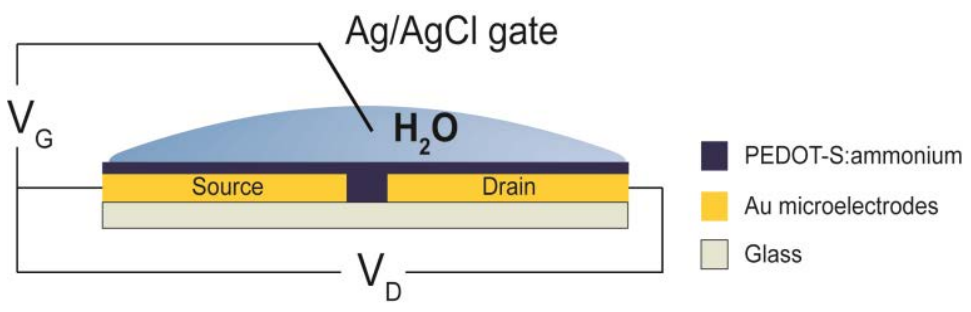

Figure 3.12 Schematic representation of an organic electrochemical transistor device comprising a water-based electrolyte $(1 \mathrm{M} \mathrm{KCl})$ and $\mathrm{Ag} / \mathrm{AgCl}$ as gate electrode.

As discussed in the previous sections, PEDOT-S:ammonium devices exhibit very high stability towards cycling, high transconductance (in the $\mathrm{mS}$ range) and fast switching times, rendering it suitable candidates for practical applications in printed electronics and bioelectronics.

\subsection{OECTs and Bioelectronics}

Organic electrochemical transistors have proved to be suitable devices for biological applications. ${ }^{33}$ Stability in aqueous environment is one of the main characteristics that render OECTs compatible with biological systems. Moreover, these devices can be fabricated on a variety of substrates and using different techniques, rendering possible to match the manufacturing processes with the desired application. Their versatility is combined with the high amplification, which is essential for the readout of biological processes. OECTs function at very low voltages $(<1 \mathrm{~V})$, which can be reduced even further by tuning the channel geometry, as shown by Malliaras et al., ${ }^{43}$ or by modifying the properties of the active material, as demonstrated in Paper 2. Operate at gate voltages around zero is desirable for applications where prolonged use of biases may lead to damaging effects, like in the case of in vivo applications.

OECTs capability of translating flows of ions from the gate to the active material into flow of electrons/holes within the active material can be used as a means to transduce biological events into electronic ones, as we will see in chapter 5 . In fact, communication in biological systems such as cells is based on ion processes and, in particular, on the transfer of ions across biomembranes, as we will see in the next chapter. 


\section{Chapter 4}

\section{Electronic Membranes}

Biological membranes or biomembranes are defined as structural elements dedicated to develop or contain certain organs, and to segregate certain fluids. The most classical example of biomembrane is the cellular membrane. The main functions of the cellular membrane are to isolate the cell from the outward environment and to exchange information, substances and energy with the outside. The main focus of this chapter is to investigate the physical structure of model biomembranes, and to demonstrate that self-doped conjugated polyelectrolytes can be added as a functional element, able either to report changes in model biomembranes organization or to provide model biomembranes and cell membranes with new functionalities.

\subsection{Biomembranes Constituents}

Cell membranes are made of many components, which vary depending on the exact nature of the cell. In 1972 a fluid-mosaic model was proposed, describing cell membranes as a phospholipid double layer, where proteins and cholesterol are embedded and all the components are free to move laterally within the biomembrane. In the recent years, the model has been revised and new concepts have been included to better describe biomembranes dynamics. ${ }^{53}$ Due to biomembranes complexity, simpler model systems have been developed to study their working mechanism. ${ }^{54}$ The advantage resides on the great precision in which the shape, the size, and the composition can be precisely controlled. Model systems are not only a useful means of study, but can be also used to deliver information to living cells, as we will see in section 4.5 .

\subsubsection{Phospholipids - Biomembranes Building Blocks}

The principal components of biomembranes are phospholipids, which are organized to form a bilayer structure. Phospholipids are amphipathic molecules, composed of a hydrophilic head (which contains a phosphate group) and two hydrophobic tails. When amphipathic molecules are 
dissolved in a polar solvent, such as water, they spontaneously selfassemble to form supramolecular structures. The hydrophilic heads maximize their interactions with polar water molecules, while their hydrophobic tails interact with each other, minimizing their interactions with the water phase. The resulting geometry will depend both on the shape of the amphipathic molecules and on the balance between hydrophilic and hydrophobic domains. As phospholipids have a "truncated cone" shape, in water solution they will self-assemble to form vesicles called liposomes (Figure 4.1). ${ }^{55}$
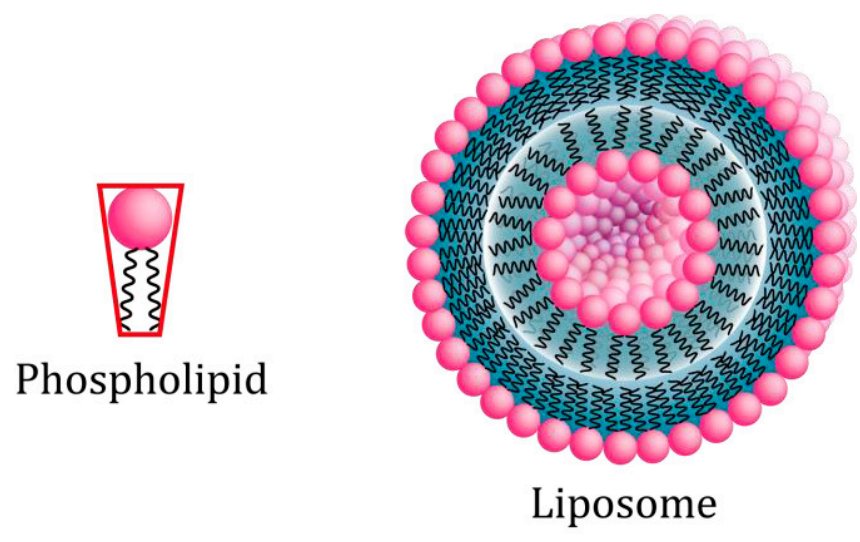

Figure 4.1 Representative picture of a phospholipid having truncated cone shape, and self-assembly of several phospholipids to form a liposome.

Liposomes are generally classified in base of their lamellarity, meaning the number of bilayers composing the liposome, and in base of their size Figure 4.2). ${ }^{56}$

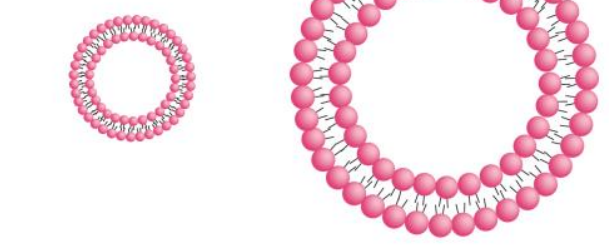

SUV 20-100 nm LUV 100-1000 nm

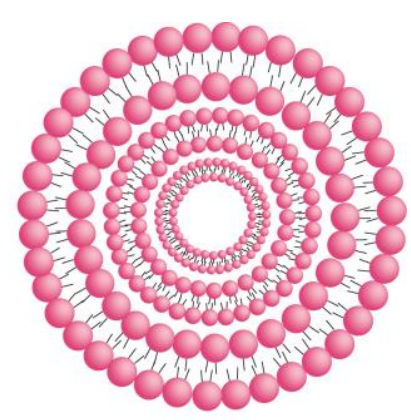

LMV 0.5-5 $\mu \mathrm{m}$

Figure 4.2 Representative picture of liposomes having different dimensions and lamellarity. The reported dimensions are average values. 
Large multilamellar vesicles (LMV) are composed by several lipid bilayers separated by water layers (Figure 4.3). They can be produced by hydration of a dried lipid film, which is accomplished by addition of an aqueous media (e.g. distilled water or buffer solution) followed by agitation.

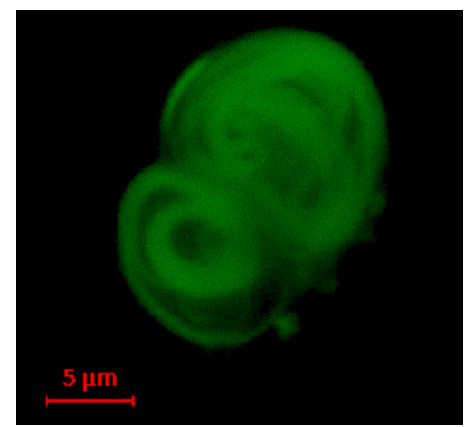

Figure 4.3 Confocal image of large multilamellar vesicles made of DOPC phospholipid (see below) and comprising 10\% (PTHS:PEDOT-S 1:3):(Oct) ${ }_{2} \mathrm{NH}_{2}$ as a fluorescent membrane probe.

Smaller vesicles sizes can be formed by disruption of LMV by sonication or extrusion. Sonication leads to the production of small unilamellar vesicles (SUV) having dimensions of $<100 \mathrm{~nm} \cdot{ }^{57}$ Extrusion is used to produce large unilamellar vesicles (LUV) having diameter $0.1 \mu \mathrm{m}-1.0$ $\mu \mathrm{m} .{ }^{58}$ A suspension of LMV is forced through a filter (polycarbonate membrane) having defined pore size. The resulting LUV will have a final diameter similar or slightly larger than the filter pore size. Giant unilamellar vesicles (GUV) having a diameter $25-100 \mu \mathrm{m}$ can also be prepared by several methods depending on the experimental conditions. ${ }^{59}$ In this thesis gentle hydration has been chosen as a preparation method due to its simplicity and no requirement of special equipment. ${ }^{60}$ However, for successful GUV preparation by this method, small amounts of a charged phospholipid (such as POPG, 1-palmitoyl-2-oleoyl-sn-glycero-3-phospho(1'-rac-glycerol) (sodium salt)) should be added to stabilize vesicles formation.

Another important parameter used to classify biomembranes is their physical state. This depends first on the kind of phospholipids composing the biomembrane. Each phospholipid is characterized by a parameter called "phase transition temperature" or "melting temperature" $\mathrm{T}_{\mathrm{m}}$, which is defined as the temperature at which the physical state of the lipid undergo a transition from solid ordered-gel phase $\left(\mathrm{S}_{\text {ord }}\right)$ to liquid disordered $\left(\mathrm{L}_{\mathrm{dis}}\right)$ state. The $\mathrm{T}_{\mathrm{m}}$ primarily depends 
on the number of unsaturated C-C bonds in the lipid alkyl chains, but also on hydrocarbon length, headgroup specie and charge. For example, 1,2dihexadecanoyl-sn-glycero-3-phosphocholine (DPPC), 1-palmitoyl-2oleoyl-sn-glycero-3-phosphocholine (POPC), and 1,2-dioleoyl-sn-glycero3-phosphocholine (DOPC) have two, one and zero unsaturated C-C bonds in their alkyl chains, respectively. Therefore, DPPC has a phase transition temperature of $41^{\circ} \mathrm{C}$, POPC of $-2^{\circ} \mathrm{C}$, and DOPC of $-17^{\circ} \mathrm{C}$ (Figure 4.4).

a)<smiles>CCCCCCCCCCCCCCCCCCCCCCC(=O)OC[C@H](COP(=O)([O-])OCC[N+](C)(C)C)OC(=O)CCCCCCCC</smiles>

b)

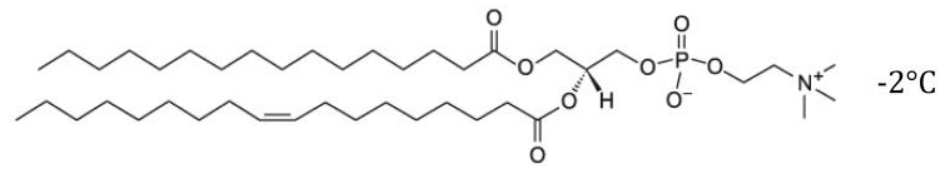

c)

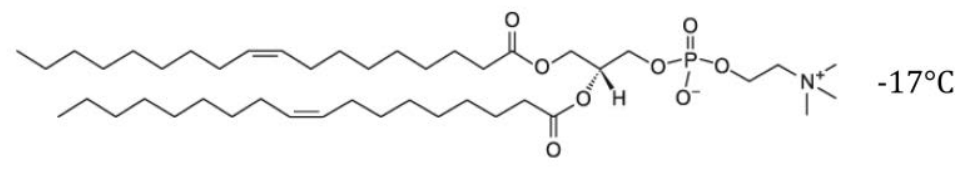

Figure 4.4 Chemical structure of a) DPPC, b) POPC, and c) DOPC phospholipids.

\subsubsection{Cholesterol - Regulator of Biomembrane Fluidity}

Another factor effecting the state of the biomembrane is the presence of small molecules in the double layer, such as cholesterol (Figure 4.5).

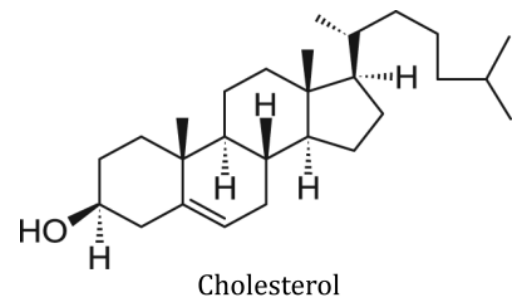

Figure 4.5 Chemical structure of cholesterol.

Presence of cholesterol leads to formation of a crystalline liquid ordered phase ( $\mathrm{L}_{\text {ord }}$ ). The main role of cholesterol in biomembranes is to maintain their fluidity upon temperature fluctuations. As a consequence, for cholesterol contents higher than a certain percentage, biomembranes will 
be in a Lord state independently of temperature, as shown in DPPC phase diagram (Figure 4.6). ${ }^{61}$

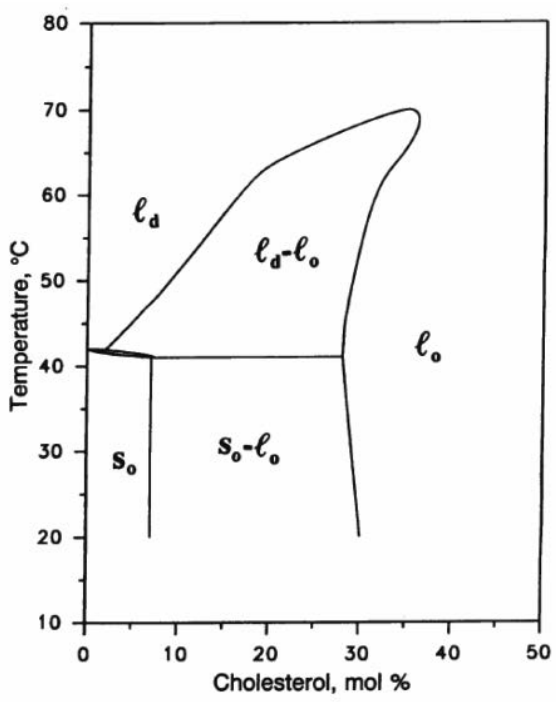

Figure 4.6 Phase diagram representing the temperature vs cholesterol content of the biomembrane. The biomembrane was composed of cholesterol and DPPC phospholipid. Reproduced with permission from 61 .

Moreover, cholesterol exhibits different affinity for different phospholipids. This can lead, in certain conditions of temperature and composition, to the coexistence of macroscopic domains. For example, three components model membranes made of DOPC:DPPC:cholesterol display coexistence of $\mathrm{L}_{\mathrm{dis}}$ and $\mathrm{L}_{\mathrm{ord}}$ phases, due to a major affinity of cholesterol for DPPC. ${ }^{62}$ Domain coexistence has great implications in more complex biological systems such as cells membranes, where they are found to have a role in regulating proteins function. ${ }^{63}$ However, mechanistic insights of the role of the so-called lipid rafts in biomembranes is still matter of debate. ${ }^{64}$ Intense effort has, therefore, been placed in developing new methods able to investigate formation of phase separated domains in biological membranes. ${ }^{65}$ In one hand, efforts have been spent in improving microscopy techniques in order to obtain sub-micrometers images. ${ }^{66}$ In the other hand, production of new fluorescent probes able to sense membranes organization is imperative to provide qualitative information as well as image membranes physical state. ${ }^{67}$ 


\subsection{Probes for Membranes Physical State}

Hydrophobic membrane probes have been introduced for the first time in 1979 by Gregorio Weber, with the synthesis of Laurdan (6-lauroyl,1-2dimethylamino naphthalene). ${ }^{68}$ Most of the existing probes are small molecules whose working mechanism is based on dipolar relaxation. ${ }^{69}$ Molecules such as Laurdan possess, in fact, a dipole moment, which increases upon excitation. If the probe is surrounded by polar molecules, such as water, reorientation of the surrounding solvent dipoles causes a decrease of the energy of the excited state of the probe, which leads to a shift of Laurdan emission maximum at higher wavelengths (red shift, Figure 4.7).

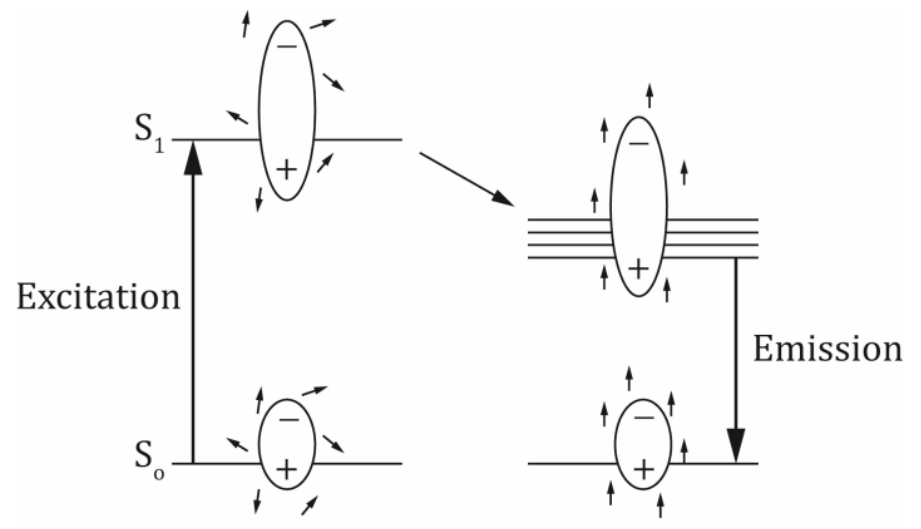

Figure 4.7 Schematic representation of dipolar relaxation in polarity sensitive probes.

If the probe is located in a $\mathbf{S}_{\text {ord }}$ phase (such as DPPC at $25^{\circ} \mathrm{C}$ ), the tight packing of the membrane prevents water molecules to enter into the lipid bilayer, therefore, no or little probe relaxation occurs. Conversely, when the probe is located in $\mathbf{L}_{\text {dis }}$ phase (such as DOPC at $25^{\circ} \mathrm{C}$ or DPPC at 55 ${ }^{\circ} \mathrm{C}$ ), more solvent molecules can penetrate into the lipid double layer. As a consequence, a red shift in emission is observed, due to a decrease of the energetic gap between excited and ground states.

Other probes can be found whose mechanism is not based on dipolar relaxation; for example, mechanosensitive membrane probes. ${ }^{70}$ Belonging to the latter class of probes, oligothiophenes have been introduced as probes whose sensing mechanism is based on both polarization and backbone planarization. ${ }^{71,72}$ The latter mechanism is based on changes in rotational freedom of the conjugated backbone in different environments, which have an impact on the conjugation length, as shown in section 2.6.1. If the probe is located in $S_{\text {ord }}$ phase, the tight packing limits the rotational freedom of the conjugated backbone, and 
leads to higher conjugation length. Phase transitions from a $S_{\text {ord }}$ to a $\mathrm{L}_{\mathrm{dis}}$ phase results in blue shifts of the emission spectra, together with modifications in the vibrational fine structure. This kind of mechanism apply also for conjugated polyelectrolytes.

\subsection{Conjugated Polyelectrolytes as Membrane Probes}

Conjugated polyelectrolytes have been tested in paper 5 as membrane probes. In contrast with previously described probes, CPEs are highly water soluble. As a consequence, CPEs are commonly added to biomembranes from water solution. ${ }^{73-75}$ A certain degree of internalization, due to the hydrophobic nature of the conjugated backbones, guarantees sensing of biomembranes physical state, as a result of changes in CPEs backbone planarization. In Paper 5 it has been shown that blends of two conjugated polyelectrolytes (PTHS and PEDOTS) combine the sensitivity provided by PEDOT backbone and the emission properties provided by PTHS. As described in section 2.7.2, the blends can be rendered hydrophobic by counterion exchange with dioctylammonium salts. Blends hydrophobicity has been exploited for incorporation in phospholipids double layers of liposome model membranes. Direct observation of the biomembranes physical state has been rendered possible by changes in absorption spectra of the blends. Variations in emission spectra have also been recorded, rendering the blends suitable candidates for imaging applications (Figure 4.8).
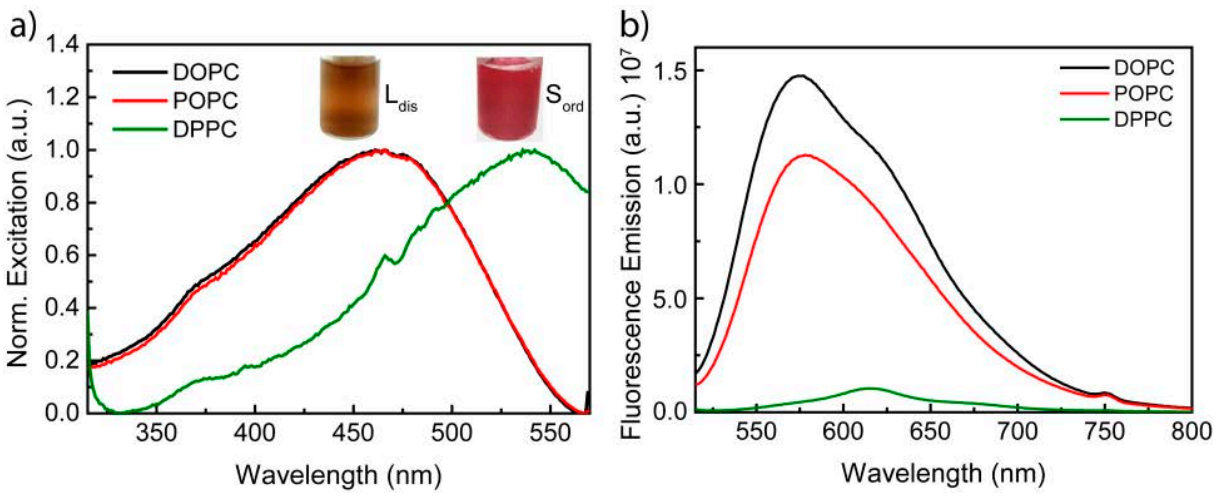

Figure 4.8 a) Excitation and b) emission spectra of blend (PTHS:PEDOT-S 1:3):(Oct) ${ }_{2} \mathrm{NH}_{2}$ incorporated into liposomes composed of DOPC (black), POPC (red), and DPPC (green).

Similar optical effects have been observed for blends in DPPC liposomes, where increase of temperature above the phase transition temperature 
leads to blue shifts in absorption and emission maxima, as described in section 4.1.1. In order to evaluate blends ability to simultaneously report phase separation in vesicles, giant unilamellar vesicles have been produced containing the blend (PTHS:PEDOT-S 1:3):(Oct) ${ }_{2} \mathrm{NH}_{2}$. GUV made of DOPC:DPPC:cholesterol, containing 10\% POPG, have been prepared as described in section 4.1.2.

The produced GUVs have been studied using confocal microscopy. Two channels, having two different excitation and acquisition wavelengths, have been simultaneously used in order to differentiate between $\mathrm{L}_{\mathrm{dis}}$ phase (signal acquired $<555 \mathrm{~nm}$, green) and $\mathrm{L}_{\text {ord }}$ phase (signal acquired $>$ $600 \mathrm{~nm}$, red), Figure 4.9.
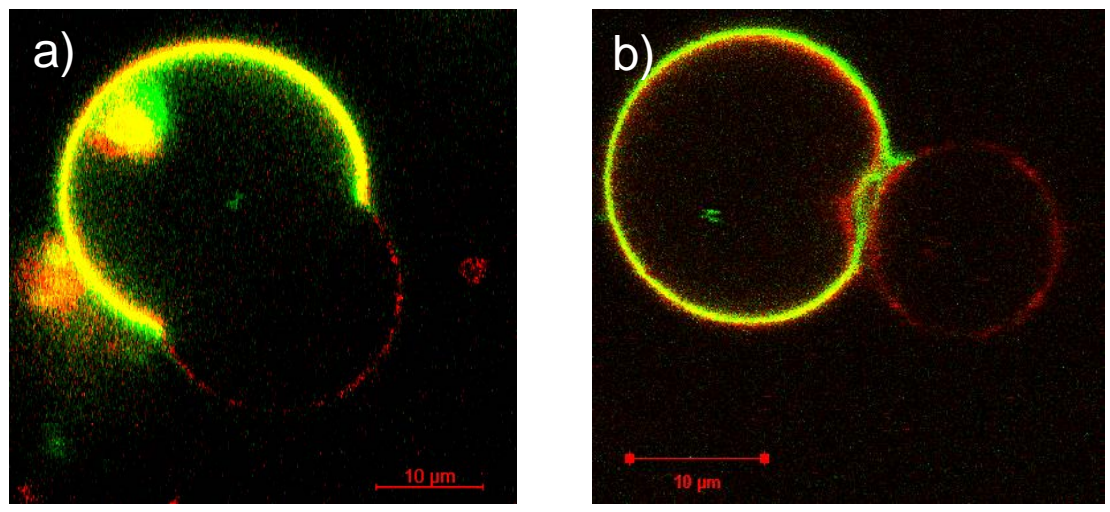

Figure 4.9 Confocal images of the blend (PTHS:PEDOT-S 1:3):(Oct) ${ }_{2} \mathrm{NH}_{2}$ incorporated into a) a two-phases GUV exhibiting coexistence of a $\mathrm{L}_{\text {dis }}$ phase (green-yellow) and Lord phase (red). b) Two GUVs displaying different Ldis and Lord phases. Excitation wavelengths: 488 $\mathrm{nm}$ for the green channel and $555 \mathrm{~nm}$ for the red channel.

The images have been useful to validate the potential of CPEs blends as probes of biomembranes organization, and exclude preferential partition of the probe into a specific phase. This work has the potential to be exploited for both probing biomembranes organization and validation of the physical state of synthetic biomembranes. ${ }^{76}$

\subsection{Biomembranes and Ion Transport}

Another component of biomembranes is represented by membrane proteins. These proteins are embedded into the phospholipid double layer and provide biomembranes with certain functionalities, such as recognition and transport. Ion channels constitute a special class of proteins able to transport ions across the membranes as a response of an 
external input. For voltage-gated ion channels the input is a change in voltage across the membrane of the cell (Figure 4.10).

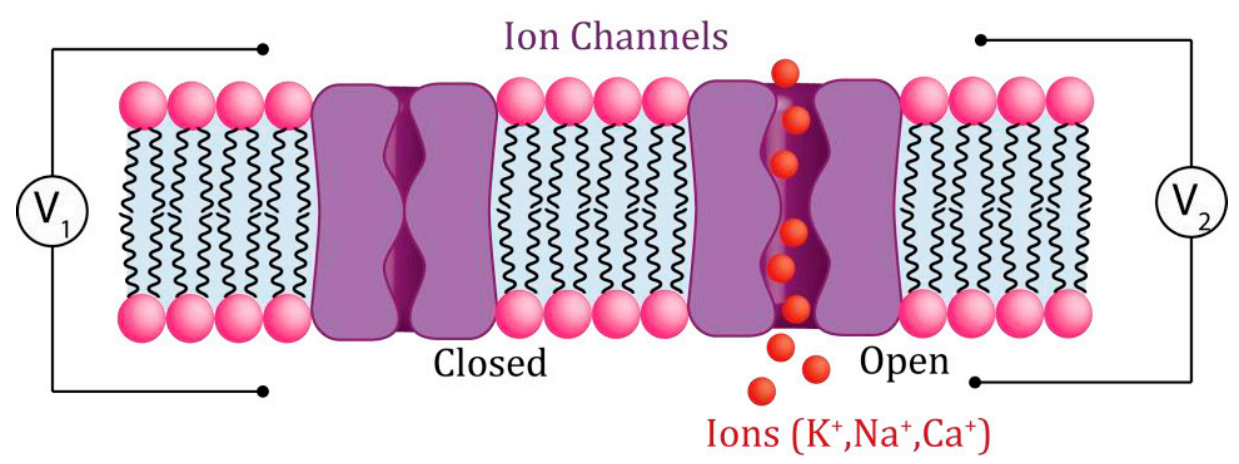

Figure 4.10 Schematic representation of closed (left) and open (right) ion channels.

Every kind of channel is specifically designed for a certain ionic specie, and it is expressed in the plasma membrane of cells. Voltage-gated ion channels, such as potassium channels, ${ }^{77}$ play a key role in the working mechanism of different kinds of cells, such as neural cells, cardiac cells, and muscle cells. Therefore, to be able to connect functions of ion channels to electronics at the nanoscale is a great opportunity to produce active interfaces between the biological and the electronic worlds. ${ }^{78}$ However, many technical difficulties have somehow prevented a straightforward connection between the two. The major challenge resides in the different ways in which the information are processed in the two worlds. Biological systems use ions, while electronics rely on electrons for information exchanges. ${ }^{79}$

\subsection{CPEs as an Interface to Ion Channels}

So far, the most explored way to interface with ion transport across biological membranes has been to produce synthetic nanopores and nanochannels able to mimic the behavior of biological channels. Synthetic systems would, in fact, offer simpler pathways to alter the transport of ions across biomembranes. ${ }^{80,81}$ In Paper 5, we aim to offer a different approach, by interfacing biological voltage-gated ion channels with an electronic conductor located inside the membrane double layer. It has been previously reported that CPEs can be introduced in biomembranes double layers, and used to modify cells surface charge. ${ }^{82}$

PEDOT-S:(Oct) ${ }_{2} \mathrm{NH}_{2}$ preserves PEDOT-S electrical conductivity while being soluble in hydrophobic solvents, as shown in section 2.7.2. In Paper 
2, this combination of properties has been exploited for the production of water-based OECTs. In Paper 5 the same material has been selected for incorporation into model membrane systems. The produced liposomes have been used as a means for the delivery of PEDOT-S:(Oct ${ }_{2} \mathrm{NH}_{2}$ to biomembranes of Xenopus oocytes. Expression of Shaker $\mathrm{K}^{+}$channels on oocytes membranes allowed to evaluate the effects of PEDOT-S:(Oct) ${ }_{2} \mathrm{NH}_{2}$ on channels activity. The two-electrode voltage clamp method has been used to record the transmembrane current as function of the applied voltage, showing increase of currents upon opening of ion channels. Results have demonstrated that PEDOT-S:(Oct) ${ }_{2} \mathrm{NH}_{2}$ produces a shift at lower potentials of ion channels opening (Figure 4.11,a). A doseresponse dependence has been observed for increased amounts of PEDOT-S:(Oct) ${ }_{2} \mathrm{NH}_{2}$ (Figure 4.11,b).
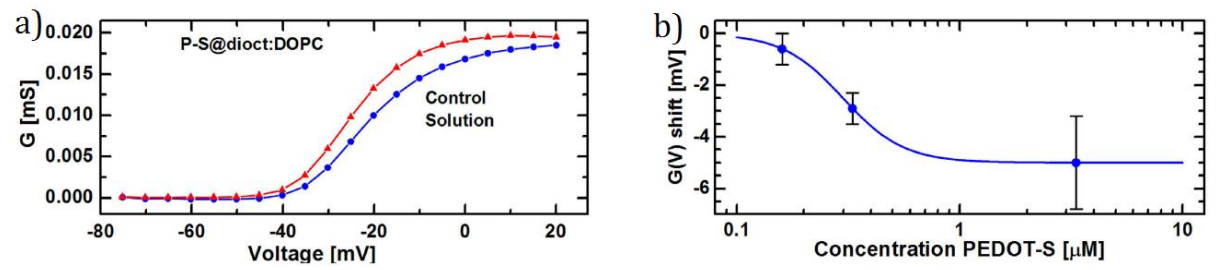

Figure 4.11 a) Steady state measurements of $\mathrm{K}^{+}$conductance $(\mathrm{G})$ as function of membrane voltage. b) Dose-response behavior of the observed conductance shift for increasing PEDOT-S:(Oct) ${ }_{2} \mathrm{NH}_{2}$ concentrations. Reproduced with permission from ${ }^{83}$.

This work has opened up for new communication routes with biological systems that can be exploited in different ways, as will be demonstrated in the next chapter. 


\section{Chapter 5}

\section{Bioelectrochemical Transistor}

The potential of organic electronics devices as tools for the study and monitoring of biological systems, as well as for providing them with external stimuli has been discussed in chapter 3.6. Among electronic devices, OECTs are extremely suitable for incorporation with biosystems. They operate at very low voltages, enabling to interface with cells and tissues in aqueous environments. The polymeric active materials can be easily functionalized as well as deposited on desired substrates, making it possible to match devices mechanical properties with the softness of tissues. Moreover, in OECTs the mixed electronic/ionic conductivity of the polymeric materials is fully exploited, rendering these devices an optimal platform for interface electronics with biology. In the previous chapters it has been shown that the same conjugated polyelectrolytes can be used for two very different purposes: to produce tunable and highly stable active materials for OECTs fabrication, and to provide biomembranes with luminescence or electronic conductivity. The aim of this chapter is to show how these two concepts can be combined, leading to new opportunities to interface electronics to the biological reign.

\subsection{Biomembranes and Cells in OECTs}

As shown in the previous chapter, biomembranes are electronic insulators. Therefore, until now they could only be integrated into OECTs as impedance components. First reports on the topic date back to 2006, where a lipid bilayer has been inserted as a blocking layer between the gate and the channel to control the gating of the OECT. ${ }^{84}$ However, the main drawback to this method is the poor stability of black lipid membranes, which limit their use for long term recordings (Figure 5.1). ${ }^{85}$ 


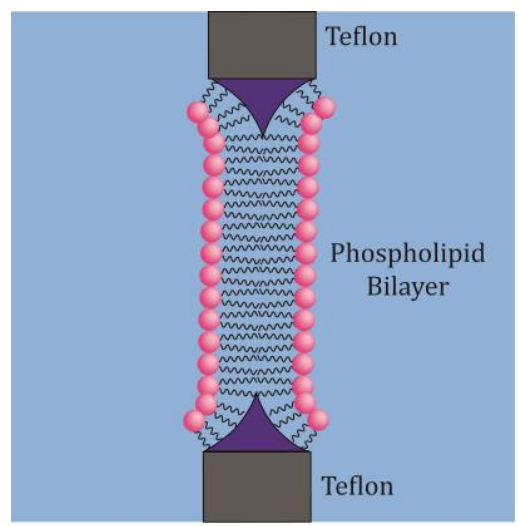

Figure 5.1 Schematic representation of a black lipid membrane. The lipid bilayer is formed across a plastic (teflon) hole, separating two chambers filled with water.

Recently, partial formation of supported lipid bilayers (SLBs) has been achieved by vesicles fusion on top of PEDOT:PSS based OECTs. ${ }^{86}$ The advantage with respect to the previous method is the higher stability of SLBs. Inclusion of ion channels into the SLBs lead to the possibility to use OECTs as sensing platforms for the recording of transmembrane proteins activity. Further advances involved the introduction of cells and tissues into the OECTs geometry. PEDOT:PSS biocompatibility enabled the culture of cells directly on top of the active material. OECTs have been successfully used to monitor barrier tissues integrity, as well as impedance sensors useful for toxicology studies. ${ }^{87-89}$ Recently, PEDOT:PSS based OECTs have been exploited for monitoring action potentials from cardiac cells, expanding the range of signals that can be recorded with these devices. ${ }^{90}$

\subsection{Preparation of Supported Lipid Bilayers (SLBs)}

Supported lipid bilayers (SLBs) are model membranes systems used for the study of the chemical and physical properties of biomembranes. Different from liposomes, SLBs possess a planar geometry and sit on a substrate (Figure 5.2). 


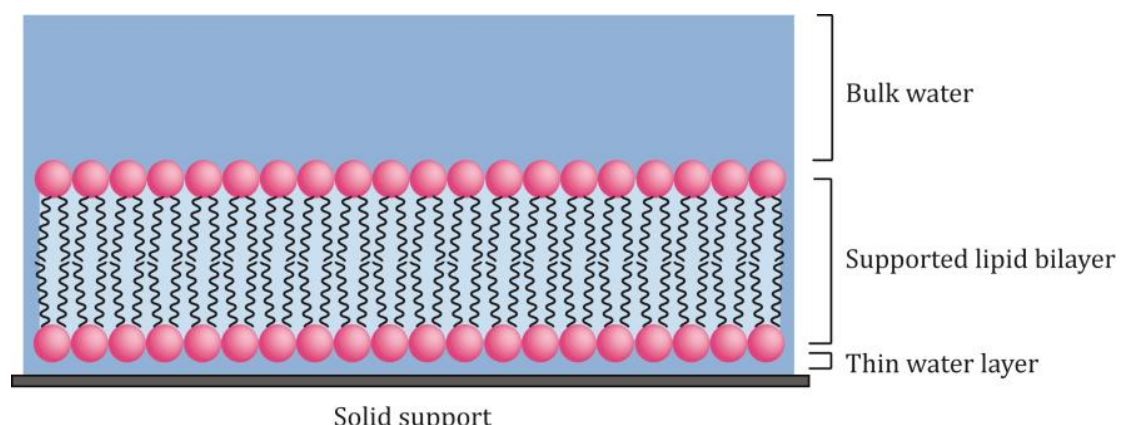

Figure 5.2 Schematic representation of a supported lipid bilayer (SLB) formed on top of a solid support.

Several methods can be used for the preparation of SLBs on various substrates. ${ }^{91}$ As previously mentioned in section 5.1, a popular method to prepare SLBs involves the spreading and fusion of SUVs on the desired substrate. ${ }^{92}$ Even though this method is conceptually simple, it presents practical issues when it comes to OECTs fabrication. The reason is that the existence of the lipid bilayer is strictly correlated to the presence of water. As we saw in chapter 4, biomembranes form by self-assembly of phospholipids in presence of water. Removal of water causes the delamination of the lipid bilayer, and loss of its supramolecular structure. ${ }^{93}$ Therefore, once hydrated, the SLB shall remain immersed in water all the time to preserve its properties. Formation of SLB by vesicles spreading on top of an OECT, therefore, requires a microfluidic setup. Moreover, successful formation of a SLB by this method is connected to careful choice of several parameters such as kind of substrate, lipids composition, presence of cations etc. Another method consists in spincoating lipids from organic solvents, and subsequently hydrate the film with the desired buffer or electrolyte. ${ }^{28,94,95}$ This method can be used on a variety of substrates and has been successfully used in Paper 6 for the production of multilayers on top of interdigitated microelectrodes arrays on glass (IMEA).

\subsection{Electronic Membranes}

In the previous chapter it has been shown that a hydrophobic electronic conductor can be inserted into the lipid bilayer of liposomes or cells to provide them with electrical conductivity. In Paper 6 this concept has been applied to supported lipid bilayers.

Cyclic voltammetry has been previously exploited in chapter 2 to study CPEs in film form. The same technique can be used to study the 
electrochemical activity of supported lipid bilayers deposited on top of a glassy carbon working electrode. The method is based on the presence of an electroactive specie in solution (like potassium ferricyanide), whose electrochemical reaction is monitored. The lipid bilayer acts as a blocking layer, preventing the electroactive specie to reach the electrode surface and the reaction to occur. When an electronic conductor is added into the phospholipid bilayer, it acts as an intermediate between the redox specie and the electrode, allowing the redox processes to occur. The charge transport ability of the electronic conductor is then monitored by the magnitude of the currents produced by interconversion of the redox specie in solution. These measurements have been used to evaluate the charge transport ability of DOPC bilayers comprising increasing amounts of PEDOT:S:(Oct) ${ }_{2} \mathrm{NH}_{2}$ (Figure 5.3).
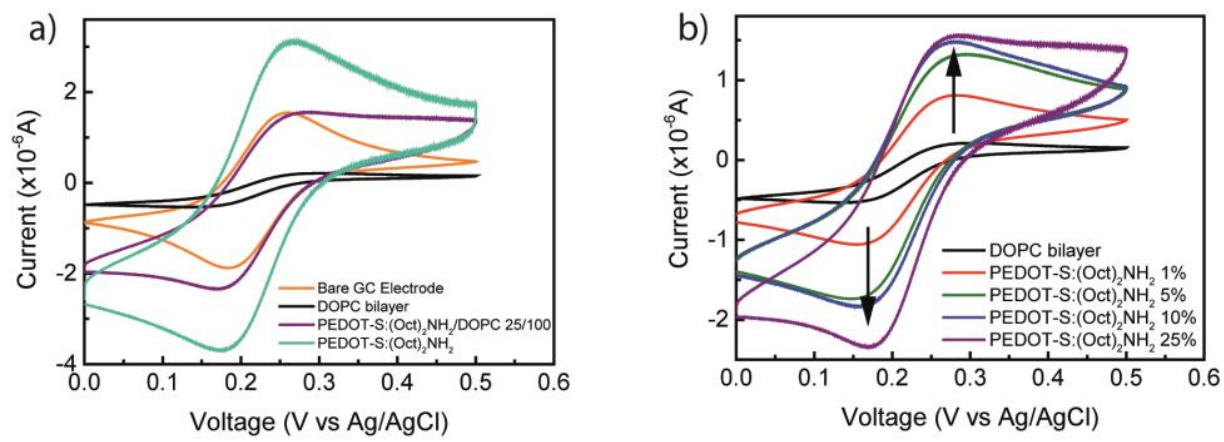

Figure 5.3 Voltammetric characterization of a) DOPC SLB formed on top of glassy carbon electrode (black), with respect to bare GC (orange), PEDOT-S:(Oct) ${ }_{2} \mathrm{NH}_{2}$ film (green), and PEDOT-S:(Oct) ${ }_{2} \mathrm{NH}_{2} /$ DOPC 25/100 SLB, and b) DOPC SLB containing 0\%, 1\%, 5\%, 10\%, and $25 \%$ of PEDOT-S:(Oct) ${ }_{2} \mathrm{NH}_{2}$.

The data provided indication of increased charge transport ability of the biomembrane in presence of increased concentrations of the electronic conductor. However, the transport of charges can be due also to formation of synthetic ion-channels by the CPEs chains aggregation. ${ }^{96}$ Electrochemical measurements using a 4-terminal setup have been performed, as described in section 2.5, to exclude the pure ion-channel behavior of the biomembranes. As no intermediate is required to perform the measurements, the only currents recorded derives from oxidation of the electroactive specie within the biomembrane (Figure 5.4). 


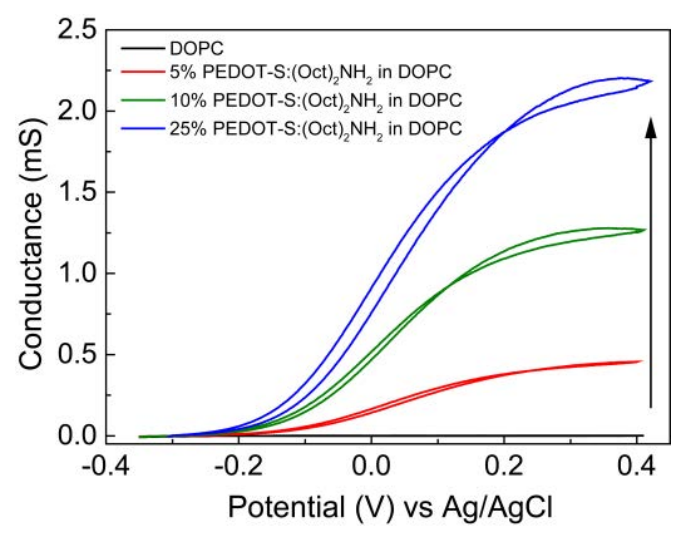

Figure 5.4 Four-electrodes conductance measurements of SLBs deposited on top of IMEAs and containing $0 \%, 5 \%, 10 \%$, and $25 \%$ of PEDOT-S:(Oct) ${ }_{2} \mathrm{NH}_{2}$.

The conductive chains shall, therefore, be close enough in space to allow the hopping of charges. The conductance thus increases as a function of concentration. The measurements clearly demonstrate the conductance properties of the biomembrane, which is not an insulating element anymore.

\subsection{A Bioelectrochemical Transistor}

Preparation of biomembranes possessing an electronic conductor lead us to the fantastic opportunity to build a fully integrated bioelectrochemical transistor (bio-ECT) comprising electronic biomembranes as active materials (Figure 5.5).

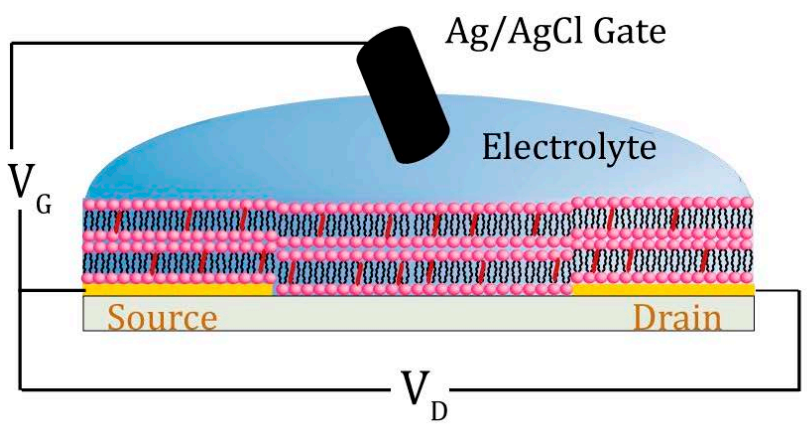

DOPC

I PEDOT:(Oct) ${ }_{2} \mathrm{NH}_{2}$

$\mathrm{Au}$ microelectrodes

Glass

Figure 5.5 Schematic representation of a bioelectrochemical transistor. The number of bilayers is indicative. 
Bio-ECTs characterization show that the devices work at very low $V_{D}$ and exhibit currents in the $\mathrm{mA}$ range. Moreover, the maximum transconductance $\mathrm{g}_{\mathrm{m}, \max }=1 \mathrm{mS}$ is quite high considering that the PEDOT:S:(Oct) ${ }_{2} \mathrm{NH}_{2}$ concentration is only $0.2 \mathrm{~g} / \mathrm{L}$ (Figure 5.6 , a). All the devices show a clear switching behavior, indicating that the $\mathrm{K}^{+}$ions can efficiently penetrate/leave the biomembrane (Figure 5.6, b).
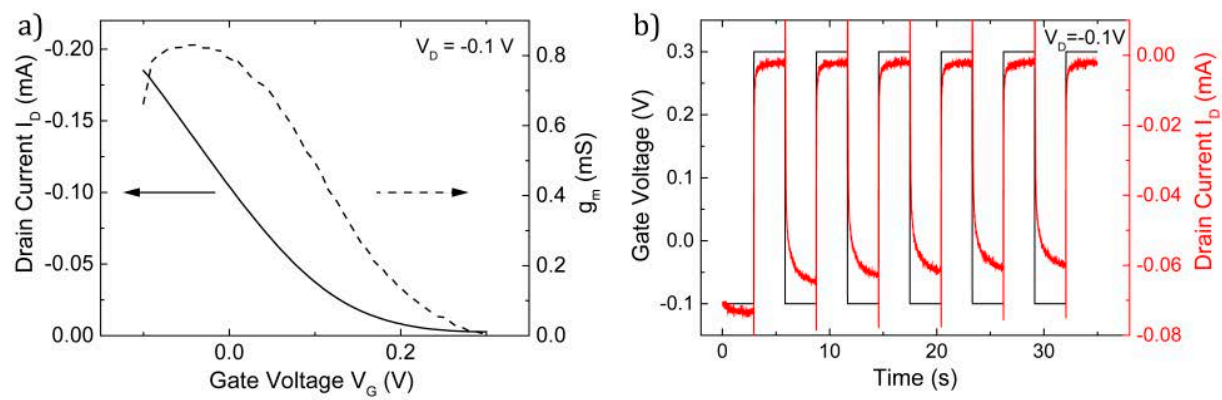

Figure 5.6 a) Transfer and b) switching characteristics of the bioelectrochemical transistor acquired for $V_{D}=-0.1 \mathrm{~V}$.

The device possess all the properties of an electrochemical transistor, with the main difference that the electronic conductor is integrated within a biomembrane. This element can help us to build new soft interfaces with biological systems, such as cells.

\subsubsection{Monitor Ion Channels in Oocytes}

In chapter 4 it has been shown that PEDOT:S:(Oct ${ }_{2} \mathrm{NH}_{2}$ can be used to modify the activity of ion channels. In this chapter the bioelectrochemical transistor has been used to monitor the expression of ion channels into the cell membrane of Xenopus oocytes. This has been accomplished by placing an oocyte on top of an electrochemical transistor and monitoring the device response (Figure 5.7). 


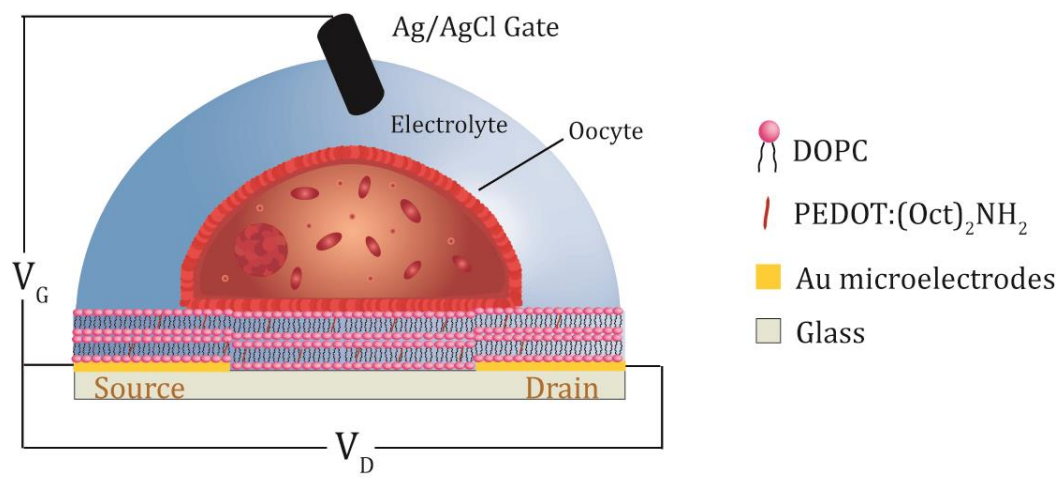

Figure 5.7 Schematic representation of a bioelectrochemical transistor with a Xenopus oocyte placed on top.

Devices transfer curves recorded in presence and absence of an oocyte on top show very big differences in drain currents (Figure 5.8, a). These results may be due to the addition of an impedance element on top of the electronic membrane, preventing ions to reach the active material, as well as a merging of oocyte and devices biomembranes and consequent dilution of the electronic conductor. Transfer curves have also been acquired in presence of an oocyte with/without overexpressed $\mathrm{K}^{+}$-gated ion channels into its cell membranes. In this case oocytes expressing ion channels showed higher currents, probably due to the restored flux of ions from the gate to the active material (Figure 5.8, b).
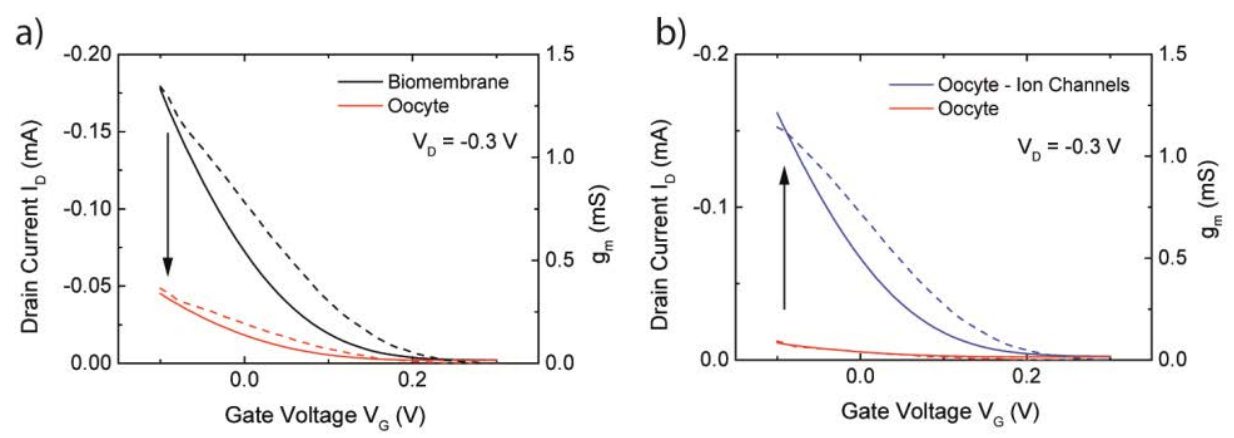

Figure 5.8 Transfer curves (solid) and related transconductance (dash) for bioelectrochemical transistors a) without (black) and with (red) oocyte on top, and b) with oocyte without (red) and with (blue) overexpressed $\mathrm{K}^{+}$ion channels. 


\section{Chapter 6}

\section{Conclusions and Future Outlook}

This thesis has been focused on self-doped conjugated polyelectrolytes. Self-assembly strategies have been used to modify the physical-chemical properties of synthesized CPEs, while preserving desired features, such as conductivity. The produced materials have then been exploited for two very different purposes.

Papers 1, 2 and 3 have been mainly focused on the production of active materials for OECTs. CPEs have been used to prepare thin films with the desired optical, physical-chemical and electronic properties. In one hand, blending two CPEs possessing different optical and electronic properties allowed fine tuning of OECTs operation mode, as well as the production of films with the optical characteristics suitable for electrochromic application. In the other hand, counterion exchange allowed both to explore blending from different solvents and to deposit a CPE from a hydrophobic solvent. The produced devices exhibit unprecedented cycling stability and gave the opportunity to incorporate an aqueous electrolyte as device component.

In papers 4 and 5 hydrophobic CPEs and CPEs blends could be integrated into biomembranes providing them with new functionalities. Blending two CPEs possessing luminescence and environmental sensitivity, has been exploited in paper 5 to produce new hydrophobic probes able to report changes in biomembranes organization. In paper 4, a hydrophobic $\mathrm{CPE}$ has been used to deliver electronic conductivity to cell membranes, and modify the activity of $\mathrm{K}^{+}$gated ion channels. Integration of an electronic conductor within biological membranes and demonstrating interaction with voltage gated ion channels opened up for several opportunities of interface electronics with biology.

In the last chapter of the thesis, and in paper 6, the lessons learned from these two different projects have been merged in order to produce electrochemical transistors integrating electronic biomembranes as channel components. The bioelectrochemical transistors represent the first example of device whose working mechanism is based on an 
electronic conductor located within the biomembrane. The produced bioECT could be successfully exploited to monitor the presence of ion channels into biomembranes of cells. With this work, a new communication pathway has been introduced between electronics and biology, which can be exploited both as a sensing platform and as a tool to study/modify biomembranes components.

For the future it would be very interesting to exploit further the possibility of tuning CPEs and CPs properties by synthetic strategies as well as self-assembly. In one hand, tuning materials properties would offer the possibility to play with fabrication conditions and lead to a better understanding of the basic principles of devices operation. In the other hand, materials engineering will offer the possibility to tailor devices characteristics for the desired application.

Another part of this work has been focused on the integration of CPEs within biomembranes. In particular, ion channels are important machines for the correct functioning of our body, and to being able to control/influence their mechanism is a step forward in the use of organic electronics as a biomedical tool. The future efforts shall be spent in understanding CPEs/channels interaction, as well as optimizing CPEs physical-chemical properties in order to render them more specific for the desired target.

The overall vision behind the last part of this work is that incorporation of biological elements within devices will lead one step closer to the use of bioelectronics in healthcare; the so called electroceuticals. ${ }^{97}$ Intimate connection between electronics and biology has the future goal to interface technology with diagnostics and therapeutics, as well as produce better platforms for drugs screening. 


\section{References}

(1)

Asghari, F.; Samiei, M.; Adibkia, K.; Akbarzadeh, A.; Davaran, S. Biodegradable and biocompatible polymers for tissue engineering application: a review. Artificial Cells, Nanomedicine, and Biotechnology 2016, 1-8.

Maitz, M. F. Applications of synthetic polymers in clinical medicine. Biosurface and Biotribology 2015, 1, 161-176.

(3)

Balint, R.; Cassidy, N. J.; Cartmell, S. H. Conductive polymers: Towards a smart biomaterial for tissue engineering. Acta Biomaterialia 2014, 10, 2341-2353.

Mayer, A. C.; Scully, S. R.; Hardin, B. E.; Rowell, M. W.; McGehee, M. D. Polymer-based solar cells. Materials Today 2007, 10, 2833.

Burroughes, J. H.; Bradley, D. D. C.; Brown, A. R.; Marks, R. N.; Mackay, K.; Friend, R. H.; Burns, P. L.; Holmes, A. B. Light-emitting diodes based on conjugated polymers. Nature 1990, 347, 539-541.

(6)

Inganas, 0. Hybrid electronics and electrochemistry with conjugated polymers. Chem. Soc. Rev. 2010, 39, 2633-2642.

(7) Pauling, L.; Brockway, L. O. Carbon-Carbon Bond Distances. The Electron Diffraction Investigation of Ethane, Propane, Isobutane, Neopentane, Cyclopropane, Cyclopentane, Cyclohexane, Allene, Ethylene, Isobutene, Tetramethylethylene, Mesitylene, and Hexamethylbenzene. Revised Values of Covalent Radii. Journal of the American Chemical Society 1937, 59, 1223-1236.

(8)

Bredas, J. L.; Street, G. B. Polarons, bipolarons, and solitons in conducting polymers. Accounts of Chemical Research 1985, 18, 309315.

Bubnova, O.; Khan, Z. U.; Wang, H.; Braun, S.; Evans, D. R.; Fabretto, M.; Hojati-Talemi, P.; Dagnelund, D.; Arlin, J.-B.; Geerts, Y. H.; Desbief, S.; Breiby, D. W.; Andreasen, J. W.; Lazzaroni, R.; Chen, W. M.; Zozoulenko, I.; Fahlman, M.; Murphy, P. J.; Berggren, M.; Crispin, X. Semimetallic polymers. Nat Mater 2014, 13, 190-194.

(10) Terao, J.; Wadahama, A.; Matono, A.; Tada, T.; Watanabe, S.; Seki, S.; Fujihara, T.; Tsuji, Y. Design principle for increasing charge mobility of $\pi$-conjugated polymers using regularly localized molecular orbitals. Nature Communications 2013, 4, 1691. 
(11)

Mikhnenko, O. V.; Cordella, F.; Sieval, A. B.; Hummelen, J. C.; Blom, P. W. M.; Loi, M. A. Temperature Dependence of Exciton Diffusion in Conjugated Polymers. The Journal of Physical Chemistry B 2008, 112, 11601-11604.

Rosu, C.; Kleinhenz, N.; Choi, D.; Tassone, C. J.; Zhang, X.; Park, J. O.; Srinivasarao, M.; Russo, P. S.; Reichmanis, E. Protein-Assisted Assembly of $\pi$-Conjugated Polymers. Chemistry of Materials 2016, 28, 573-582.

Alemu, D.; Wei, H.-Y.; Ho, K.-C.; Chu, C.-W. Highly conductive PEDOT:PSS electrode by simple film treatment with methanol for ITOfree polymer solar cells. Energy \& Environmental Science 2012, 5, 96629671.

Patil, A. O.; Ikenoue, Y.; Basescu, N.; Colaneri, N.; Chen, J.; Wudl, F.; Heeger, A. J. Self-doped conducting polymers. Synthetic Metals 1987, 20, 151-159.

(15) Qiao, Q.; Beck, J.; McLeskey, J. J. T. In Photovoltaic devices from self-doped polymers, 2005; 2005; pp 59380E-59380E-59389.

(16) Tran-Van, F.; Carrier, M.; Chevrot, C. Sulfonated polythiophene and poly(3,4-ethylenedioxythiophene) derivatives with cations exchange properties. Synthetic Metals 2004, 142, 251-258.

Tóth, P. S.; Samu, G. F.; Endrődi, B.; Visy, C. Hyphenated in situ conductance and spectroelectrochemical studies of polyaniline films in strongly acidic solutions. Electrochimica Acta 2013, 110, 446-451.

Gao, Y.; Wang, C.-C.; Wang, L.; Wang, H.-L. Conjugated Polyelectrolytes with pH-Dependent Conformations and Optical Properties. Langmuir 2007, 23, 7760-7767.

Knaapila, M.; Evans, R. C.; Garamus, V. M.; Almásy, L.; Székely, N. K.; Gutacker, A.; Scherf, U.; Burrows, H. D. Structure and "Surfactochromic" Properties of Conjugated Polyelectrolyte (CPE): Surfactant Complexes between a Cationic Polythiophene and SDS in Water. Langmuir 2010, 26, 15634-15643.

(20) Neo, W. T.; Ye, Q.; Chua, S.-J.; Xu, J. Conjugated polymerbased electrochromics: materials, device fabrication and application prospects. Journal of Materials Chemistry C 2016, 4, 7364-7376.

Kaim, W.; Fiedler, J. Spectroelectrochemistry: the best of two worlds. Chem. Soc. Rev. 2009, 38, 3373-3382.

(22) Burrows, H. D.; Knaapila, M.; Fonseca, S. M.; Costa, T. Conjugated Polyelectrolytes; Wiley-VCH Verlag GmbH \& Co. KGaA: 2012.

(23) Brendel, J. C.; Schmidt, M. M.; Hagen, G.; Moos, R.; Thelakkat, M. Controlled Synthesis of Water-Soluble Conjugated Polyelectrolytes Leading to Excellent Hole Transport Mobility. Chem. Mater. 2014, 26, 1992-1998. 
Heeley, M. E. H.; Gallaher, J. K.; Nguyen, T. L.; Woo, H. Y.; Hodgkiss, J. M. Surfactant controlled aggregation of conjugated polyelectrolytes. Chemical Communications 2013, 49, 4235-4237.

(25) Pace, G.; Friend, R. Conjugated Polyelectrolytes; Wiley-VCH Verlag GmbH \& Co. KGaA: Weinheim, 2012.

(26) Pan, C.; Sugiyasu, K.; Takeuchi, M. Blending conjugated polymers without phase separation for fluorescent colour tuning of polymeric materials through FRET. Chemical Communications 2014, 50, 11814-11817.

(27) Benten, H.; Mori, D.; Ohkita, H.; Ito, S. Recent research progress of polymer donor/polymer acceptor blend solar cells. Journal of Materials Chemistry A 2016, 4, 5340-5365.

(28) Pompeo, G.; Girasole, M.; Cricenti, A.; Cattaruzza, F.; Flamini, A.; Prosperi, T.; Generosi, J.; Congiu Castellano, A. AFM characterization of solid-supported lipid multilayers prepared by spin-coating. Biochimica et Biophysica Acta (BBA) - Biomembranes 2005, 1712, 29-36.

(29) Andersson Ersman, P.; Nilsson, D.; Kawahara, J.; Gustafsson, G.; Berggren, M. Fast-switching all-printed organic electrochemical transistors. Organic Electronics 2013, 14, 1276-1280.

(30) Berggren, M.; Nilsson, D.; Robinson, N. D. Organic materials for printed electronics. Nat. Mater. 2007, 6, 3-5.

(31) Bihar, E.; Deng, Y.; Miyake, T.; Saadaoui, M.; Malliaras, G. G.; Rolandi, M. A Disposable paper breathalyzer with an alcohol sensing organic electrochemical transistor. Scientific Reports 2016, 6, 27582.

(32) Scheiblin, G.; Aliane, A.; Strakosas, X.; Curto, V. F.; Coppard, R.; Marchand, G.; Owens, R. M.; Mailley, P.; Malliaras, G. G. Screen-printed organic electrochemical transistors for metabolite sensing. MRS Communications 2015, 5, 507-511.

(33) Strakosas, X.; Bongo, M.; Owens, R. M. The organic electrochemical transistor for biological applications. Journal of Applied Polymer Science 2015, 132, n/a-n/a.

(34) Mabeck, J. T.; Malliaras, G. G. Chemical and biological sensors based on organic thin-film transistors. Analytical and Bioanalytical Chemistry 2006, 384, 343-353.

White, S. P.; Dorfman, K. D.; Frisbie, C. D. Operating and Sensing Mechanism of Electrolyte-Gated Transistors with Floating Gates: Building a Platform for Amplified Biodetection. The Journal of Physical Chemistry C 2016, 120, 108-117.

(36) Tarabella, G.; Santato, C.; Yang, S. Y.; Iannotta, S.; Malliaras, G. G.; Cicoira, F. Effect of the gate electrode on the response of organic electrochemical transistors. Applied Physics Letters 2010, 97, 123304. 
Stavrinidou, E.; Leleux, P.; Rajaona, H.; Khodagholy, D.; Rivnay, J.; Lindau, M.; Sanaur, S.; Malliaras, G. G. Direct Measurement of Ion Mobility in a Conducting Polymer. Advanced Materials 2013, 25, 4488-4493.

Inal, S.; Rivnay, J.; Leleux, P.; Ferro, M.; Ramuz, M.; Brendel, J. C.; Schmidt, M. M.; Thelakkat, M.; Malliaras, G. G. A High Transconductance Accumulation Mode Electrochemical Transistor. Advanced Materials 2014, 26, 7450-7455.

(39)

Bernards, D. A.; Malliaras, G. G. Steady-State and Transient Behavior of Organic Electrochemical Transistors. Advanced Functional Materials 2007, 17, 3538-3544.

Giovannitti, A.; Nielsen, C. B.; Sbircea, D.-T.; Inal, S.; Donahue, M.; Niazi, M. R.; Hanifi, D. A.; Amassian, A.; Malliaras, G. G.; Rivnay, J.; McCulloch, I. N-type organic electrochemical transistors with stability in water. Nature Communications 2016, 7, 13066.

Panzer, M. J.; Frisbie, C. D. Polymer Electrolyte-Gated Organic Field-Effect Transistors: Low-Voltage, High-Current Switches for Organic Electronics and Testbeds for Probing Electrical Transport at High Charge Carrier Density. Journal of the American Chemical Society 2007, 129, 6599-6607.

(42) Nielsen, C. B.; Giovannitti, A.; Sbircea, D.-T.; Bandiello, E.; Niazi, M. R.; Hanifi, D. A.; Sessolo, M.; Amassian, A.; Malliaras, G. G.; Rivnay, J.; McCulloch, I. Molecular Design of Semiconducting Polymers for HighPerformance Organic Electrochemical Transistors. Journal of the American Chemical Society 2016, 138, 10252-10259.

Rivnay, J.; Leleux, P.; Sessolo, M.; Khodagholy, D.; Hervé, T.; Fiocchi, M.; Malliaras, G. G. Organic Electrochemical Transistors with Maximum Transconductance at Zero Gate Bias. Advanced Materials 2013, 25, 7010-7014.

(44) Khodagholy, D.; Gurfinkel, M.; Stavrinidou, E.; Leleux, P.; Herve, T.; Sanaur, S.; Malliaras, G. G. High speed and high density organic electrochemical transistor arrays. Appl. Phys. Lett. 2011, 99, 163304.

Friedlein, J. T.; Donahue, M. J.; Shaheen, S. E.; Malliaras, G. G.; McLeod, R. R. Microsecond Response in Organic Electrochemical Transistors: Exceeding the Ionic Speed Limit. Advanced Materials 2016, $28,8398-8404$.

(46) Deyu, T.; Nilsson, D.; Forchheimer, R. Electrochromic Electrochemical Transistors Gated With Polyelectrolyte-Decorated Amyloid Fibrils. Display Technology, Journal of 2013, 9, 755-759. Andersson, P.; Forchheimer, R.; Tehrani, P.; Berggren, M. Printable All-Organic Electrochromic Active-Matrix Displays. Advanced Functional Materials 2007, 17, 3074-3082. 
(48)

Li, Y.; Qian, R. Stability of conducting polymers from the electrochemical point of view. Synthetic Metals 1993, 53, 149-154.

(49) Gaupp, C. L.; Zong, K.; Schottland, P.; Thompson, B. C.; Thomas, C. A.; Reynolds, J. R. Poly(3,4-ethylenedioxypyrrole): Organic Electrochemistry of a Highly Stable Electrochromic Polymer. Macromolecules 2000, 33, 1132-1133.

(50) Zhang, S.; Kumar, P.; Nouas, A. S.; Fontaine, L.; Tang, H.; Cicoira, F. Solvent-induced changes in PEDOT:PSS films for organic electrochemical transistors. APL Mater. 2015, 3, 014911.

(51) Rivnay, J.; Inal, S.; Collins, B. A.; Sessolo, M.; Stavrinidou, E.; Strakosas, X.; Tassone, C.; Delongchamp, D. M.; Malliaras, G. G. Structural control of mixed ionic and electronic transport in conducting polymers. Nature Communications 2016, 7, 11287.

(52) Zhang, S.; Hubis, E.; Girard, C.; Kumar, P.; DeFranco, J.; Cicoira, F. Water stability and orthogonal patterning of flexible microelectrochemical transistors on plastic. Journal of Materials Chemistry $C$ 2016, 4, 1382-1385.

Nicolson, G. L. The Fluid-Mosaic Model of Membrane Structure: Still relevant to understanding the structure, function and dynamics of biological membranes after more than 40 years. Biochimica et Biophysica Acta (BBA) - Biomembranes 2014, 1838, 1451-1466.

(54) Chan, Y.-H. M.; Boxer, S. G. Model Membrane Systems and Their Applications. Current opinion in chemical biology 2007, 11, 581-587. (55) Akbarzadeh, A.; Rezaei-Sadabady, R.; Davaran, S.; Joo, S. W.; Zarghami, N.; Hanifehpour, Y.; Samiei, M.; Kouhi, M.; Nejati-Koshki, K. Liposome: classification, preparation, and applications. Nanoscale Research Letters 2013, 8, 102-102.

(56) Laouini, A.; Jaafar-Maalej, C.; Limayem-Blouza, I.; Sfar, S.; Charcosset, C.; Fessi, H. Preparation, Characterization and Applications of Liposomes: State of the Art. Journal of Colloid Science and Biotechnology 2012, 1, 147-168.

(57) Klingler, J.; Vargas, C.; Fiedler, S.; Keller, S. Preparation of ready-to-use small unilamellar phospholipid vesicles by ultrasonication with a beaker resonator. Analytical Biochemistry 2015, 477, 10-12.

(58) MacDonald, R. C.; MacDonald, R. I.; Menco, B. P. M.; Takeshita, K.; Subbarao, N. K.; Hu, L.-r. Small-volume extrusion apparatus for preparation of large, unilamellar vesicles. Biochimica et Biophysica Acta (BBA) - Biomembranes 1991, 1061, 297-303.

(59) Walde, P.; Cosentino, K.; Engel, H.; Stano, P. Giant Vesicles: Preparations and Applications. ChemBioChem 2010, 11, 848-865. 
(60)

Akashi, K.; Miyata, H.; Itoh, H.; Kinosita, K. Preparation of giant liposomes in physiological conditions and their characterization under an optical microscope. Biophysical Journal 1996, 71, 3242-3250.

Sankaram, M. B.; Thompson, T. E. Cholesterol-induced fluidphase immiscibility in membranes. Proceedings of the National Academy of Sciences of the United States of America 1991, 88, 8686-8690.

(62) Engberg, O.; Hautala, V.; Yasuda, T.; Dehio, H.; Murata, M.; Slotte, J. P.; Nyholm, Thomas K. M. The Affinity of Cholesterol for Different Phospholipids Affects Lateral Segregation in Bilayers. Biophysical Journal 2016, 111, 546-556.

Lucero, H. A.; Robbins, P. W. Lipid rafts-protein association and the regulation of protein activity. Archives of Biochemistry and Biophysics 2004, 426, 208-224.

(64) Kaiser, H.-J.; Lingwood, D.; Levental, I.; Sampaio, J. L.; Kalvodova, L.; Rajendran, L.; Simons, K. Order of lipid phases in model and plasma membranes. Proceedings of the National Academy of Sciences 2009, 106, 16645-16650.

(65) Jacobson, K.; Mouritsen, O. G.; Anderson, R. G. W. Lipid rafts: at a crossroad between cell biology and physics. Nat Cell Biol 2007, 9, 714.

Bates, M.; Jones, S. A.; Zhuang, X. Stochastic optical reconstruction microscopy (STORM): a method for superresolution fluorescence imaging. Cold Spring Harbor protocols 2013, 2013, 498-520.

Klymchenko, Andrey S.; Kreder, R. Fluorescent Probes for Lipid Rafts: From Model Membranes to Living Cells. Chemistry \& Biology 2014, 21, 97-113.

Weber, G.; Farris, F. J. Synthesis and spectral properties of a hydrophobic fluorescent probe: 6-propionyl-2(dimethylamino)naphthalene. Biochemistry 1979, 18, 3075-3078.

Sezgin, E.; Sadowski, T.; Simons, K. Measuring Lipid Packing of Model and Cellular Membranes with Environment Sensitive Probes. Langmuir 2014, 30, 8160-8166.

(70) Dal Molin, M.; Verolet, Q.; Soleimanpour, S.; Matile, S. Mechanosensitive Membrane Probes. Chemistry - A European Journal 2015, 21, 6012-6021.

Dal Molin, M.; Matile, S. 3,4-Ethylenedioxythiophene in planarizable push-pull oligothiophenes. Organic \& Biomolecular Chemistry 2013, 11, 1952-1957.

Dal Molin, M.; Verolet, Q.; Colom, A.; Letrun, R.; Derivery, E.; Gonzalez-Gaitan, M.; Vauthey, E.; Roux, A.; Sakai, N.; Matile, S. Fluorescent Flippers for Mechanosensitive Membrane Probes. Journal of the American Chemical Society 2015, 137, 568-571. 
(73)

Feng, G.; Ding, D.; Liu, B. Fluorescence bioimaging with conjugated polyelectrolytes. Nanoscale 2012, 4, 6150-6165.

(74) Houston, J. E.; Kraft, M.; Scherf, U.; Evans, R. C. Sequential detection of multiple phase transitions in model biological membranes using a red-emitting conjugated polyelectrolyte. Physical Chemistry Chemical Physics 2016.

(75) Kahveci, Z.; Martínez-Tomé, M. J.; Mallavia, R.; Mateo, C. R. Use of the Conjugated Polyelectrolyte Poly\{[9,9-bis $\left(6^{\prime}-\mathrm{N}, \mathrm{N}, \mathrm{N}-\right.$ trimethylammonium)hexyl]fluorene-phenylene\} Bromide (HTMA-PFP) as a Fluorescent Membrane Marker. Biomacromolecules 2013, 14, 19901998.

(76) Brea, R. J.; Rudd, A. K.; Devaraj, N. K. Nonenzymatic biomimetic remodeling of phospholipids in synthetic liposomes. Proceedings of the National Academy of Sciences 2016, 113, 8589-8594.

(77) Fowler, P. W.; Sansom, M. S. P. The pore of voltage-gated potassium ion channels is strained when closed. Nature Communications 2013, 4, 1872.

(78) Fromherz, P. Three Levels of Neuroelectronic Interfacing. Annals of the New York Academy of Sciences 2006, 1093, 143-160.

(79) Simmel, F. C. Bioelectronics: Wiring-up ion channels. Nat Phys 2009, 5, 783-784.

(80) Geng, J.; Kim, K.; Zhang, J.; Escalada, A.; Tunuguntla, R.; Comolli, L. R.; Allen, F. I.; Shnyrova, A. V.; Cho, K. R.; Munoz, D.; Wang, Y. M.; Grigoropoulos, C. P.; Ajo-Franklin, C. M.; Frolov, V. A.; Noy, A. Stochastic transport through carbon nanotubes in lipid bilayers and live cell membranes. Nature 2014, 514, 612-615.

(81) Tagliazucchi, M.; Szleifer, I. Transport mechanisms in nanopores and nanochannels: can we mimic nature? Materials Today 2015, 18, 131-142.

(82) Catania, C.; Thomas, A. W.; Bazan, G. C. Tuning cell surface charge in E. coli with conjugated oligoelectrolytes. Chemical Science 2016, 7, 2023-2029.

Johansson, P. K.; Jullesson, D.; Elfwing, A.; Liin, S. I.; Musumeci, C.; Zeglio, E.; Elinder, F.; Solin, N.; Inganäs, O. Electronic polymers in lipid membranes. Scientific Reports 2015, 5, 11242.

(84) Bernards, D. A.; Malliaras, G. G.; Toombes, G. E. S.; Gruner, S. M. Gating of an organic transistor through a bilayer lipid membrane with ion channels. Applied Physics Letters 2006, 89, 053505.

(85) Jeon, T.-J.; Malmstadt, N.; Poulos, J. L.; Schmidt, J. J. Black lipid membranes stabilized through substrate conjugation to a hydrogel. Biointerphases 2008, 3, FA96-FA100. 
(86)

Zhang, Y.; Inal, S.; Hsia, C.-Y.; Ferro, M.; Ferro, M.; Daniel, S.; Owens, R. M. Supported Lipid Bilayer Assembly on PEDOT:PSS Films and Transistors. Advanced Functional Materials 2016, n/a-n/a.

(87)

Jimison, L. H.; Tria, S. A.; Khodagholy, D.; Gurfinkel, M.; Lanzarini, E.; Hama, A.; Malliaras, G. G.; Owens, R. M. Measurement of Barrier Tissue Integrity with an Organic Electrochemical Transistor. Advanced Materials 2012, 24, 5919-5923.

(88) Rivnay, J.; Ramuz, M.; Leleux, P.; Hama, A.; Huerta, M.; Owens, R. M. Organic electrochemical transistors for cell-based impedance sensing. Applied Physics Letters 2015, 106, 043301.

Tria, S. A.; Ramuz, M.; Huerta, M.; Leleux, P.; Rivnay, J.; Jimison, L. H.; Hama, A.; Malliaras, G. G.; Owens, R. M. Dynamic Monitoring of Salmonella typhimurium Infection of Polarized Epithelia Using Organic Transistors. Advanced Healthcare Materials 2014, 3, 1053-1060.

Yao, C.; Li, Q.; Guo, J.; Yan, F.; Hsing, I. M. Rigid and Flexible Organic Electrochemical Transistor Arrays for Monitoring Action Potentials from Electrogenic Cells. Advanced Healthcare Materials 2015, 4, 528-533.

El Kirat, K.; Morandat, S.; Dufrêne, Y. F. Nanoscale analysis of supported lipid bilayers using atomic force microscopy. Biochimica et Biophysica Acta (BBA) - Biomembranes 2010, 1798, 750-765.

(92) Hardy, G. J.; Nayak, R.; Zauscher, S. Model cell membranes: Techniques to form complex biomimetic supported lipid bilayers via vesicle fusion. Current Opinion in Colloid \& Interface Science 2013, 18, 448-458.

(93)

Han, C.-T.; Chao, L. Creating Air-Stable Supported Lipid Bilayers by Physical Confinement Induced by Phospholipase A2. ACS Applied Materials \& Interfaces 2014, 6, 6378-6383.

(94) Mennicke, U.; Salditt, T. Preparation of Solid-Supported Lipid Bilayers by Spin-Coating. Langmuir 2002, 18, 8172-8177.

(95) Simonsen, A. C.; Bagatolli, L. A. Structure of Spin-Coated Lipid Films and Domain Formation in Supported Membranes Formed by Hydration. Langmuir 2004, 20, 9720-9728.

(96) Zhang, Z.; Shi, J.; Huang, W. Study of the ion-channel behavior on glassy carbon electrode supported bilayer lipid membranes stimulated by perchlorate anion. Materials Science and Engineering: $C$ 2015, 55, 431-435.

(97) Famm, K.; Litt, B.; Tracey, K. J.; Boyden, E. S.; Slaoui, M. Drug discovery: A jump-start for electroceuticals. Nature 2013, 496, 159-161. 


\section{Papers}

The articles associated with this thesis have been removed for copyright reasons. For more details about these see:

http://urn.kb.se/resolve?urn=urn:nbn:se:liu:diva-132731 